IZA DP No. 6059

Five Issues in the Design of Income Support Mechanisms: The Case of Italy

Ugo Colombino

October 2011 


\title{
Five Issues in the Design of Income Support Mechanisms: The Case of Italy
}

\author{
Ugo Colombino \\ University of Turin, \\ CHILD and IZA
}

\section{Discussion Paper No. 6059 \\ October 2011}

\author{
IZA \\ P.O. Box 7240 \\ 53072 Bonn \\ Germany \\ Phone: +49-228-3894-0 \\ Fax: +49-228-3894-180 \\ E-mail: iza@iza.org
}

\begin{abstract}
Any opinions expressed here are those of the author(s) and not those of IZA. Research published in this series may include views on policy, but the institute itself takes no institutional policy positions.

The Institute for the Study of Labor (IZA) in Bonn is a local and virtual international research center and a place of communication between science, politics and business. IZA is an independent nonprofit organization supported by Deutsche Post Foundation. The center is associated with the University of Bonn and offers a stimulating research environment through its international network, workshops and conferences, data service, project support, research visits and doctoral program. IZA engages in (i) original and internationally competitive research in all fields of labor economics, (ii) development of policy concepts, and (iii) dissemination of research results and concepts to the interested public.
\end{abstract}

IZA Discussion Papers often represent preliminary work and are circulated to encourage discussion. Citation of such a paper should account for its provisional character. A revised version may be available directly from the author. 


\section{ABSTRACT}

\section{Five Issues in the Design of Income Support Mechanisms: The Case of Italy}

Differently from most European countries and despite the recommendations on the part of the European Commission, Italy still misses a sufficiently systematic and nationwide mechanism of income support. In this paper we want to explore the feasibility, the desirability and the features of a universal policy of minimum income in Italy. We use a microeconometric model and a social welfare methodology in order to evaluate various alternatives mechanisms. We simulate the effects and the social welfare performance of 30 reforms resulting from six versions of five basic types of income support mechanism: guaranteed minimum income (GMI), universal basic income (UBI), wage subsidy (WS) and two mixed systems: GMI+WS and UBI+WS. As welfare evaluation criteria we adopt the Gini Social Welfare function and the Poverty-Adjusted Gini Social Welfare function. All the reforms are calibrated so as to preserve fiscal neutrality. The simulation adopts a methodology that allows for market equilibrium and ensures a consistent comparative statics interpretation of the results. Universal and non mean-tested transfers (possibly complemented by wage subsidy) emerge as desirable and feasible features of the income support mechanism. In the most realistic scenarios, the social-welfare-optimal policies are a modest unconditional transfer amounting to $40 \%$ of the poverty line complemented by a $10 \%$ wage subsidy or depending on the social welfare criterion - a more generous unconditional transfer $(100 \%$ of the poverty line). The reforms can be financed by proportionally increasing the current marginal tax rates and widening the tax base to include all personal incomes. The set of universalistic policies that are preferable to the current system is very large and gives the opportunity of selecting a best reform according to many different criteria.

JEL Classification: C35, C53, H31, J22

Keywords: design of income support mechanisms, basic income, guaranteed minimum income, wage subsidy, discrete choice, labour supply, simulation of tax reforms, equilibrium microsimulation

Corresponding author:

Ugo Colombino

Department of Economics Cognetti de Martiis

University of Torino

Via Po 53

10124 Torino

Italy

E-mail: ugo.colombino@unito.it

\footnotetext{
* Edlira Narazani, research associate at the Department of Economics Cognetti De Martiis, has contributed to this work with skilful programming by performing all the econometric estimations and policy simulations. I wish to thank Lennart Flood and Rolf Aaberge for useful comments especially on the equilibrium simulation procedure. Help from the EUROMOD team who provided the 1998 Italian dataset and from the Compagnia di San Paolo who provided financial support is gratefully acknowledged. The usual disclaimers apply.
} 


\section{Introduction}

Differently from most European countries and despite the recommendations on the part of the EC, Italy still misses a sufficiently systematic and nationwide mechanism of income support, although various selective or conditional income maintenance policies are operating and some local authorities are experimenting forms of minimum income policy. ${ }^{2}$ In this paper we explore the feasibility, the desirability and the features of a universal policy of minimum income in Italy. The starting point is provided by optimal taxation theory, i.e. we aim at designing an income support mechanism that replaces the actual policies and maximizes a given social welfare function subject to a public budget constraint. However, instead of looking for an analytical solution we adopt a computational-empirical approach. Namely, we use a microeconometric model and a social welfare methodology in order to explore and evaluate various alternatives mechanisms. In illustrating the motivations, the methods and the results, we will refer to five issues that emerge as crucial in the analysis of reforms, whether hypothetical or implemented:

1) Is a universal income support mechanism feasible and desirable?

2) Should the mechanism consist of a transfer or a subsidy or a combination of the two? A significant part of the recent literature on the design of income support mechanisms is focussed on comparing transfer-like policies (such as the negative income tax, the demogrant, the basic income etc.) versus subsidy-like policies (such as earned income tax credit, in-work benefits etc.). The former permit the attainment of a minimum level of income through a lump-sum transfer, while the latter provide the opportunity of receiving a higher income by supporting a higher net age rate. Most numerical simulations done with the model of Mirrlees (1971) suggest as an optimal system a tax-transfer schedule with a lump-sum transfer, very high marginal tax rates on low income and almost constant marginal tax rates on average and high income. This scenario seems to have inspired many reforms (implemented or discussed) in the three decades 1970-80-90. A second scenario emerges since the end of the 90s, with contributions (e.g. Diamond (1998) and Saez $(2001,2002))$ that make Mirrlees' model more amenable to econometric applications and generalize it to include the decision of whether to work or not (not only - as in Mirrlees (1971) - the decision of how much to work). This latter extension is particularly relevant for the design of income support mechanisms. An influential contribution is represented by in particular by Saez (2002), whose model has been adopted in various applications (e.g. Immervoll et al., 2007; Haan et al., 2007; Blundell et al., 2009). A frequent result emerging from these studies is the superiority of policies such as in-work benefits, or taxcredit on low earnings. Interestingly, analogous policies have been in part implemented or considered as alternatives to mean-tested transfers in various countries during the last decade. The theoretical nature of the optimal taxation literature in practice has forced the analysis to address transfer-based and subsidy-based

\footnotetext{
${ }^{2}$ While we are writing, in the EU countries only Greece, Hungary and Italy do not implement a nation-wide minimum income policy. Since 1992 the European Commission has issued many declarations and recommendations where minimum income policies emerge as a key instrument for enforcing fundamental human rights, reducing poverty and promoting social inclusion. A useful survey of minimum income policies in Europe is provided by Busilacchi (2008).
} 
mechanisms as if they were strictly alternative. But nothing prevents the design of mechanisms that combine the two policies. In what follows we will also consider such mixed policies.

3) Should a transfer be conditional or unconditional? The transfers mentioned at point 2) are typically conditional (e.g. mean-tested). Unconditional transfers have also been proposed (universal basic income, citizen income etc.). So far the idea of a universal and unconditional transfer has never reached the position of a dominating scenario but it remains an inspiring idea with oscillating fortunes. It has strong philosophical motivations (e.g. Van Parjis 1995), but also cost-benefit and efficient incentives arguments are sometimes put forward: unconditional transfers do not incur the costs of verifying and monitoring the eligibility conditions; they do not create poverty traps; they might promote more autonomy and more efficient choices in the educational and occupational career etc. (Barrientos and Lloyd-Sherlock 2002, Standing 2008). Atkinson (2002) suggests that various processes in the modern economies might naturally drive the social policy institutions toward the universal basic income scenario.

4) How generous should the policy be? Every income support mechanism needs to specify the level of minimum income. This applies to transfer policies since they typically aim at guaranteeing that a certain minimum income is attained. But it also applies to incentive-base policies, since the subsidies are usually active only up to a certain level of income. The typical amount (in proposed or implemented reforms) is not larger than the poverty level and in most cases is much lower. This is so because the mechanisms are designed as complementary with respect to other welfare and social policies. There are however more extreme versions where the amount is supposed to be more substantial either because it is meant to replace the whole welfare state, as in Friedman (1962), or because it is thought as a fundamental political-economic restructuring of the market economy, as in Van Parijs (1995).

5) Should taxes (that also finance the income support mechanism) be progressive or flat? Universal mechanisms of income support (whether transfer-based or subsidy-based) have been frequently presented together with the proposal of a flat-tax. The motivation was to counterbalance the costs and/or the (supposedly) negative incentives coming from income support with better incentive to labour supply for the (supposedly) most productive fraction of the population. However, the above argument ignores the fact that labour supply elasticity is inversely related to income levels (Aaberge et al. 1999, 2002, 2011a, 2011b) and takes it for granted - despite the ambiguous empirical evidence - that income support mechanisms have strong negative effects on labour supply. When taking into account these empirical facts, we might be led instead to support a progressive taxation. 


\section{The alternative policies}

In this section we summarize the main features of the hypothetical tax-transfer reforms that will be simulated under the assumption that they completely replace the actual tax-transfer system (a detailed description is provided in Appendix C). They are stylized cases representative of the different scenarios that are discussed or even actually implemented in many countries. A key parameter in the definition of the policies is the threshold G defined as follows. Let

$x_{i}=$ total net available income (current) of household $i$ (including both couples and singles).

$N_{i}=$ total number of components of household i.

Define the "individual-equivalent" income: $\tilde{x}_{i}=\frac{x_{i}}{\sqrt{N_{i}}}$ and the Poverty Line $P=\frac{1}{2} \operatorname{median}(\tilde{x})$. Then $G_{i}=a P \sqrt{N_{i}}$, where $a$ is a proportion. For each reform we simulate three versions with different values of $a: 1,0.75$ and 0.50 . For example, $G=$ $0.5 P \sqrt{3}$ means that for a household with 3 components the threshold is $1 / 2$ of the Poverty Line times the equivalence scale $\sqrt{3} .^{3}$

Guaranteed Minimum Income (GMI). Each individual receives a transfer equal to $G-I$ if single or $G / 2-I$ if partner in a couple provided $I<G$ (or $I<G / 2$ ), where $I$ denotes individual taxable income. This is the standard conditional (or means-tested) income support mechanism, close to a Negative Income Tax (Friedman 1962) with a 100\% marginal tax rate on the transfer.

Universal Basic Income (UBI). Each individual receives an unconditional transfer equal to $G$ if single or $G / 2$ if partner in a couple. It is the basic version of the system discussed for example by Van Parijs (1995) and also known in the policy debate as "citizen income" or "social dividend" (Meade, 1972; Van Trier, 1995). ${ }^{4}$

Wage Subsidy (WS). Each individual receives a 10\% subsidy on the gross hourly wage and her/his income is not taxed as long as her/his gross income (including the subsidy) does not exceed $G$ if single or $G / 2$ if partner in a couple. This is close to various in-work benefits or tax-credits reforms introduced in the USA (Earned Income Tax Credit), in the UK (In-Work Benefits) and recently also in Sweden.

GMI + WS and UBI + WS are mixed mechanisms where the transfer is coupled with the wage subsidy, but with the threshold redefined as $0.5 G .^{5}$

\footnotetext{
${ }^{3}$ The "square root scale" is one of the equivalence scales commonly used in OECD publications.

${ }^{4}$ A somewhat mitigated version has been proposed by Atkinson $(1995,1996)$ as Participation Income, where the transfer is conditional upon a test of "participation" (work, education, voluntary social activities, child care, homework etc.).
} 
For each of the above five types we distinguish two versions: a flat tax version, in which the income support mechanism is matched with a fixed marginal tax rate $t$ applied to individual incomes above $G$ for singles or $G / 2$ for the partners of couple; a progressive tax version, in which the income support mechanism is matched with a progressive tax (that replicates the current system but with marginal tax rates proportionally adjusted according to a constant $\tau$ ) that applies to incomes exceeding $G$ (or $G / 2$ ). The parameters $t$ and $\tau$ are endogenously determined within the reform simulation so that the total net tax revenue is equal to the one collected under the current tax-transfer system. Altogether we have 5 (types) $\times$ $3($ values of $a) \times 2($ tax rules $)=30$ reforms.

All the tax-transfer policies are individual-based. ${ }^{6}$

\section{The microeconometric model}

We develop a microeconometric model of household labour supply that is capable of simulating the household choices, taxes paid, transfers received, net available income and attained utility level given any tax-transfer rule regime, under the constraint of a constant total net tax revenue.

Appendix A provides a detailed description of the model. Here we offer an intuitive overview. Although we actually treat both couples and singles, for the sake of simplicity the following illustration considers singles.

The model assumes households are endowed with unearned income $y$ and face a set $A$ of opportunities ("jobs") characterized by hours of market work required $(h)$, gross wage rate $(w)$ and other characteristics $(\mathrm{j})$. The opportunity set includes non-market "jobs" (i.e. activities - such as child care or education - outside the labour market, with $h=0$ and therefore $w h=0$ ). Opportunity sets can differ across households, both in terms of wage rates and in terms of availability of market jobs (including the case of no market job available) with different hours and characteristics. The tax-transfer rule $\mathrm{R}$ (actual or simulated) transforms the gross incomes ( $w h, y)$ into the net available income $C$. The household preferences upon alternative jobs are represented by a utility function $U(h, C, j)$. The model assumes households choose a job so as to maximize $U(h, C, j)$ subject to the opportunity constraint $(h, w, j) \in A$ and the budget constraint $C=R(w h, y)$. Under this assumption, the observed choices reveal the household preferences and with appropriate datasets and statistical procedures it is therefore possible to estimate a parametric specification of the utility function. Once we have estimated the utility function, we can simulate what the household choices would be when facing a different opportunity set, e.g. one induced by a tax-transfer reform.

\footnotetext{
${ }^{5}$ A mixed system close to GMI+WS has been proposed by De Vincenti and Paladini (2009).

${ }^{6}$ We also simulated German-like (income splitting) household-based versions, but they were mostly dominated (in terms of welfare effects) by the individual-based ones, so we chose not to report them.
} 


\section{Social Welfare evaluation}

Since the tax-transfer reforms in general have different effects on different households we need a criterion to "aggregate" all the micro-effects into a synthetic index in order to be able to compare and evaluate the reforms. We will use two indexes. The first one is based on Sen $(1974,1976)$, who proposed to compare different statuses of the economy by computing namely $\mu(1-I)$, where $\mu$ is the average income and $I$ is the Gini coefficient of the income distribution. This measure has the intuitive appeal of expressing social welfare as the product of an efficiency measure (average income, i.e. the average size of the "pie's slices") time a familiar equality measure (1- I), i.e. a measure of how equally the "pie" is allocated among the households). We apply the same idea using money-metric utility instead of income. Let $\mu^{n}(R)$ be the maximum money-metric utility attained under tax-transfer regime $R$ by household $n$ (computed as explained in Section A.6 of Appendix A) and $\mu(R)=\frac{1}{N} \sum_{n} \mu^{n}(R)$. Let $I(R)$ be the Gini coefficient of the sample distribution of $\mu^{n}(R)$. We then define the Gini Social Welfare (GSW) function as follows: ${ }^{7}$

$G S W(R)=\mu(R)(1-I(R))$.

The second index - the Poverty-adjusted Gini Social Welfare (PAGSW) - is a generalization that gives a specific weight to poverty (Atkinson 1987):

$\operatorname{PAGSW}(R)=\mu(R)(1-I(R)-p(R))$

where $p(R)$ is the head-count poverty ratio under the tax-transfer regime $R$.

\section{Simulation procedure}

The simulation has two distinctive features that are not common in the tax reform literature. First, the reforms are simulated under the constraint of being fiscally neutral, i.e. they generate the same total net tax revenue as the current 1998 system. This requires a two-level simulation procedure. At the "low" level, household choices are simulated given the values of the tax-transfer parameters. At the "high" level, the tax-transfer parameters are calibrated so that the total net tax revenue remains constant. The calibration parameters are the constant tax rate $t$ in the Flat tax systems and the proportional change $\tau$ of the current marginal tax rates in the Progressive tax systems. ${ }^{8}$ Second, the simulation is conducted under equilibrium conditions for different hypothetical values of the elasticity of the demand for labour. Traditionally, the simulation of tax reforms are interpreted as comparative statics exercises in a long-run perspective, i.e.

\footnotetext{
${ }^{7}$ For a theoretical justification of this social welfare function (as a member of a wider class) see for example Aaberge (2007) and Aaberge et al. (2011b).

${ }^{8}$ Current (1998) marginal tax rates are reported in Appendix C.
} 
assuming a perfectly elastic labour demand (constant wage rates). At the other extreme, non-behavioural simulations can be interpreted as simulations in the very short-run. There are of course an infinity of intermediate scenarios. We adopt a procedure that is specifically appropriate for the microeconometric model and makes the simulation results consistent with the comparative statics interpretation. The procedure is fully explained in Colombino (2010) and more concisely in the Appendix B to this paper.

We perform six types of simulations, corresponding to different treatment of equilibrium:

Non behavioural. Household choices are left unchanged, while their incomes are changed according to the new taxbenefit rules. This can be considered as a prediction of the very short-run.

No account for equilibrium. This is the standard procedure. Labour supply responses are simulated while keeping wage rates unchanged. Usually this is interpreted as a long run prediction under the hypothesis of a perfectly elastic demand for labour. However, as we argue in Appendix B, this interpretation in general is not correct when adopting a model that incorporates a representation of demand condition (the multinomial logit with alternative-specific dummies).

Demand elasticity $\eta=0,-0.5,-1$. Most empirical studies of wage elasticity of the demand for labour suggest values in the range $(-0.5,-1)$.

Demand elasticity $\boldsymbol{\eta}=-\infty$. This is a theoretical benchmark. It should be interpreted as indicating the direction towards which we move if we assume a very elastic demand.

We consider as realistic scenarios those with $\eta=-0.5$ and $\eta=-1$. The other cases are reported as benchmarks.

\section{Results}

Tables 1 - 3 illustrate the main welfare evaluation results. We start by commenting the results of Tables 1 and 2 following the six-issue outline introduced in section 1. Moreover, if not otherwise indicated, we refer to the results obtained under the most realistic scenarios, i.e. $\eta=-0.5$ or -1 . The policies (30 reforms plus the current system) are ranked - the most preferred on top - according to the social welfare functions presented in section 4. Each reform is identified by three pieces of information: the income support mechanism (GMI etc.), the Flat (F) or Progressive (P) tax rule and the value of $a(0.5,0.75$ or 1$)$. For example, UBI+WS_F_0.75 denotes a policy where the income support mechanism is UBI+WS, the tax rule is Flat and $G=0.75 P \sqrt{N}$.

1) Most reforms rank better than the current system under both social welfare criteria. The only exception appears when $\eta=\infty$ and the policies are ranked according to the GSW: in this case, no reform turns out as preferred to the current system. However $\eta=\infty$ represents a benchmark case rather than a realistic scenario. In all the other cases there is a very large menu of universalistic reforms that dominate (in terms of welfare) the current system. Therefore the answer to the first issue mentioned in section 1 is definitively affirmative. As we comment below, 
the welfare criteria adopted here gives specific answers as to what mechanisms are best. However, also from the point of view of different criteria, we have many alternatives among which to choose in order to improve upon the current system.

2) In most cases, the first four or five positions in the ranking are occupied by transfer-based mechanisms or by mixed policies envisaging both transfers and subsidies. Under this respect, we observe a marked difference between the GSW criterion and the PAGSW criterion. The former criterion favours the mixed policy UBI+WS while the latter favours a pure UBI.

3) Overall, mechanisms envisaging unconditional transfers (UBI or UBI+WS) rank better than the conditional systems. The greater generosity of the unconditional transfers is compensated by the lack of poverty-trap effects, so that the conditional and the unconditional systems imply similar very modest reductions in labour supply; however, the unconditional systems perform better in favouring distributional equity and reducing poverty.

4) Under GSW, the basic transfer should be $75 \%$ of the poverty line; under the PAGSW it should be $100 \%$.

5) In most cases Progressive tax systems are preferable to Flat tax systems. A contribution to this result comes from the pattern of wage elasticity of labour supply: higher income households are much less elastic than lower income ones (Aaberge et al. 1999, 2002, 2004; Aaberge and Colombino 2011a, 2011b; Røed and Strøm 2002). ${ }^{9}$

In summary, the indications for a best mechanism converge on UBI+WS_P_0.75 (under the GSW criterion) or UBI_P_1 (under the PAGSW criterion).

In Table 3 we report the result of a regression analysis of the results obtained under the scenario with $\eta=-1$. The value of the Social Welfare function is regressed against a set of variables measuring the key features of the tax-transfer systems. The regressions help to identify the welfare contribution of policy attributes. Under the GSW criterion, the results confirm that the progressivity of the tax rule and the non-conditionality of the income support mechanism have a significant positive effect. The coverage $a$ has a positive marginal effect up to around 0.70 . The picture produced by the PAGSW criterion is partially different. Overall the coefficients are much larger, since there is much more variation in the GSW than in the PAGSW. The effects of Progressive and Unconditional are positive as under the GSW, but less significant. Instead the effect of Subsidy is negative and significant. Coverage has a positive marginal effect even above 1.

What specific features do the best mechanisms have and how do they fare from the perspective of other possibly relevant criteria, such as marginal tax rates or behavioural effects? Tables $5-10$ provide many relevant details. Here the policies are listed in alphabetic order. For each type of simulation (No Behaviour, No equilibrium etc.) and for each policy the tables report the results listed in the Legenda (Table 4). The following comments consider what happens under the scenario with $\eta=-1$ (Table 9).

\footnotetext{
${ }^{9}$ A recent survey by Diamond and Saez (2011) gives support to the superiority of progressive taxes. This conclusion might be mitigated or even reversed if one accounted for the transparency and simplicity of the tax rule, for incentives to tax elusion/evasion and in general for a more general concept of behavioural response to taxes as in the "taxable income" approach (e.g. Gruber and Saez 2002).
} 
1) UBI+WS_P_0.75 (UBI_P_1) envisages an average monthly transfer of 720 (1060) Euros (1998). This is to be compared with the 101 Euros of the CURRENT system. ${ }^{10}$ The percentages of utility-winners and of incomewinners are respectively 69 (57) and 65 (58). The percentage poverty rate (head count) is 0.9 (0), to be compared to 4.23 under the CURRENT system.

2) Typical objections against universalistic policy of income support are based on the expectation of high tax rates required by the public budget constraint and of strong disincentive effects on labour supply. The first expectation is confirmed by our results. The best (welfare-wise) policies are costly in terms of marginal tax rates.

UBI+WS_P_0.75 would require an 11\% increase of the current (1998) marginal tax rates, which means a 50\% top marginal tax rate. Under the same scenario UBI_P_1 requires a 60\% top marginal tax rate. It should be noticed that these figures are high but not at all unrealistic. For example in 2009 the top marginal tax rates in Denmark and Sweden were respectively around $62 \%$ and $57 \%$. At any rate, if the above tax rates were judged for some reasons not feasible (possibly from the point of view of political consensus), we have already noticed that the menu of welfare improving reforms is very large. For example, the flat version UBI+WS_F_0.75 would require a $42 \%$ flat rate. The second expectation (strong disincentive effects on labour supply) is not supported by our results: the overall disincentive effects are small.

3) When we account for behavioural responses and for market equilibrium, the policies turn out to be less costly (tax-wise) than when we assume no behavioural responses or we do not account for market equilibrium. In shaping the simulation results there is a subtle interplay between the behavioural responses and the market equilibrium process. Overall, the reform induce a (modest) shift to the left of the labour supply curve, therefore the new market equilibrium requires a higher gross wage rate (provided $\eta>-\infty$ ). The pure effect on taxation of the behavioural responses can be identified by compare Table 5 to Table 10 , where $\eta=-\infty$ and therefore wage rates remain unchanged. The reform UBI+WS_P_0.75 would require a $14 \%$ increase in current marginal tax rates when assuming no behavioural responses (Table 5). The same reform would instead require a $12 \%$ increase in current marginal tax rates when accounting for behaviour (but leaving wage rates unchanged). Despite the overall reduction in labour supply, the reform induces a more efficient composition of employment Last, if we assume $\eta=-1$ (Table 9), the increase would be $11 \%$ : higher gross wage help in collecting tax revenue and therefore the reform requires a lower increase in marginal tax rates.

4) Accounting for behavioural responses and market equilibrium does also have significant implications for the ranking position of the policies. The differences in ranking are more marked when the GSW criterion is used. It seems that with the PAGSW criterion the rankings are strongly influenced by the effects on the head-count poverty index, which in turn are similar across different simulation procedure: as a consequence the differences in rankings are mitigated, especially among the highest rank positions.

\footnotetext{
${ }^{10}$ It should be noticed that the 101 Euros transfer in the CURRENT system is just the average of various categorical, conditional or local transfers and benefits (such as unemployment benefits, "Cassa Integrazione", family benefits etc.).
} 


\section{Conclusions}

We used a microeconometric model and a social evaluation methodology in order to identify optimal universalistic income support mechanisms in Italy. We consider five type of mechanism: GMI, UBI, WS, GMI+WS and UBI+WS. Each one has three variants, depending on the degree of coverage with respect to the poverty line: 50\%, $75 \%$ and $100 \%$. Moreover, each type can be match either with Flat tax rule or with a Progressive Tax rule. In total we have $5 \times 3 \times 2=30$ possible reforms. The tax parameter (either constant flat rate in the Flat rule or the proportional change in the marginal tax rates with respect to the current (1998) system in the Progressive rule) is determined endogenously so that the total net tax revenue remains as under the current system. The simulation adopts a methodology (Colombino 2010) that allows for market equilibrium and ensures a consistent comparative statics interpretation of the simulation results. Accounting for behavioural responses and market equilibrium has important implications in shaping the simulation results. In the most realistic scenarios (i.e. wage elasticity of labour demand in the range $[-0.5,-1.0]$ ) the best policies are UBI+WS with a $75 \%$ coverage and a progressive tax rule (under the GSW criterion) or UBI with a 100\% coverage and a progressive tax rule (under the PAGSW criterion). Given the chosen social welfare criteria, universality, non-conditionality, progressivity, wage subsidies (under the GSW criterion) and a minimum income close to $75 \%$ of the poverty line or above, emerge as desirable features of an optimal income support mechanism. In general, the set of universalistic policies that are preferable to the current system is very large and gives the opportunity of selecting a best reform according to many different criteria. 
Table 1. Policies ranked (from best to worst) according to the GSW criterion

\begin{tabular}{|c|c|c|c|c|c|c|c|c|c|c|c|c|c|c|c|c|c|}
\hline \multicolumn{3}{|c|}{ No behaviour } & \multicolumn{3}{|c|}{ No equilibrium } & \multicolumn{3}{|c|}{ Equilibrium $\eta=0$} & \multicolumn{3}{|c|}{ Equilibrium $\eta=-\mathbf{0 . 5}$} & \multicolumn{3}{|c|}{ Equilibrium $\eta=-1$} & \multirow{2}{*}{\multicolumn{3}{|c|}{$\begin{array}{c}\text { Equilibrium } \boldsymbol{\eta}=\mathbf{- o o} \\
\text { CURRENT }\end{array}$}} \\
\hline UBI+WS & $\mathrm{P}$ & & UBI+WS & $\mathrm{P}$ & 1 & GMI & $\mathrm{F}$ & 1 & UBI+WS & $\mathrm{P}$ & 0.75 & UBI+WS & & 0.75 & & & \\
\hline UBI+WS & $\mathrm{P}$ & 0.75 & UBI & $\mathrm{P}$ & 0.75 & GMI & $\mathrm{F}$ & 0.75 & UBI & $\mathrm{P}$ & 0.5 & UBI & $\mathrm{P}$ & 0.5 & UBI+WS & $\mathrm{P}$ & 0.5 \\
\hline UBI & $P$ & 0.75 & $\mathrm{UBI}+\mathrm{WS}$ & $\mathrm{P}$ & 0.75 & UBI & $\mathrm{F}$ & 0.75 & WS & $\mathrm{P}$ & 0.75 & UBI & $\mathrm{P}$ & 0.75 & GMI+WS & $\mathrm{P}$ & 0.5 \\
\hline UBI & $\mathrm{P}$ & 0.5 & UBI & $\mathrm{P}$ & 0.5 & GMI & $\mathrm{P}$ & 1 & $\mathrm{UBI}+\mathrm{WS}$ & $\mathrm{P}$ & 0.5 & WS & $\mathrm{P}$ & 0.75 & UBI & $\mathrm{F}$ & 0.5 \\
\hline WS & $\mathrm{P}$ & 1 & UBI & $\mathrm{P}$ & 1 & UBI & $\mathrm{F}$ & 0.5 & UBI & $\mathrm{P}$ & 0.75 & UBI+WS & $\mathrm{P}$ & 0.5 & UBI & $\mathrm{P}$ & 0.5 \\
\hline UBI & $\mathrm{P}$ & 1 & WS & $\mathrm{P}$ & 1 & GMI & $\mathrm{P}$ & 0.75 & WS & $\mathrm{P}$ & 0.5 & WS & $\mathrm{P}$ & 0.5 & WS & $\mathrm{P}$ & 0.5 \\
\hline UBI+WS & $\mathrm{P}$ & 0.5 & $\mathrm{UBI}+\mathrm{WS}$ & $\mathrm{P}$ & 0.5 & UBI & $\mathrm{P}$ & 0.5 & UBI & $\mathrm{P}$ & 1 & UBI & $\mathrm{P}$ & 1 & WS & $\mathrm{F}$ & 0.5 \\
\hline WS & $P$ & 0.75 & WS & $\mathrm{P}$ & 0.75 & UBI+WS & $\mathrm{F}$ & 1 & GMI+WS & $\mathrm{P}$ & 0.5 & GMI+WS & $\mathrm{P}$ & 0.5 & GMI+WS & $\mathrm{F}$ & 0.5 \\
\hline WS & $\mathrm{P}$ & 0.5 & WS & $\mathrm{P}$ & 0.5 & UBI & $F$ & 1 & GMI+WS & $\mathrm{P}$ & 0.75 & GMI+WS & $\mathrm{P}$ & 0.75 & UBI+WS & $\mathrm{F}$ & 0.5 \\
\hline UBI & $\mathrm{F}$ & 1 & UBI & $\mathrm{F}$ & 1 & GMI & $\mathrm{P}$ & 0.5 & WS & $\mathrm{F}$ & 1 & WS & $\mathrm{P}$ & 1 & GMI & $\mathrm{P}$ & 0.5 \\
\hline UBI & $\mathrm{F}$ & 0.75 & UBI & $\mathrm{F}$ & 0.75 & GMI & $\mathrm{F}$ & 0.5 & WS & $\mathrm{P}$ & 1 & WS & $\mathrm{F}$ & 1 & GMI & $\mathrm{F}$ & 0.5 \\
\hline GMI+WS & $P$ & 1 & GMI+WS & $\mathrm{P}$ & 1 & UBI+WS & $\mathrm{F}$ & 0.75 & UBI & $\mathrm{F}$ & 1 & UBI & $\mathrm{F}$ & 0.75 & UBI+WS & $\mathrm{P}$ & 0.75 \\
\hline GMI+WS & $P$ & 0.75 & GMI+WS & $\mathrm{P}$ & 0.75 & GMI+WS & $\mathrm{F}$ & 1 & UBI & $\mathrm{F}$ & 0.75 & UBI & $\mathrm{F}$ & 1 & GMI+WS & $\mathrm{P}$ & 0.75 \\
\hline GMI+WS & $\mathrm{P}$ & 0.5 & GMI+WS & $\mathrm{P}$ & 0.5 & UBI & $\mathrm{P}$ & 0.75 & UBI+WS & $\mathrm{F}$ & 1 & UBI+WS & $\mathrm{F}$ & 1 & WS & $\mathrm{F}$ & 0.75 \\
\hline WS & $F$ & 1 & UBI+WS & $F$ & 1 & GMI+WS & $\mathrm{F}$ & 0.75 & UBI & $\mathrm{F}$ & 0.5 & UBI & $\mathrm{F}$ & 0.5 & GMI+WS & $F$ & 0.75 \\
\hline UBI+WS & $F$ & 1 & UBI & $F$ & 0.5 & UBI+WS & $\mathrm{P}$ & 0.5 & WS & $\mathrm{F}$ & 0.75 & GMI & $\mathrm{P}$ & 0.5 & WS & $P$ & 0.75 \\
\hline UBI & $F$ & 0.5 & $\mathrm{UBI}+\mathrm{WS}$ & $F$ & 0.75 & UBI+WS & $\mathrm{P}$ & 0.75 & GMI & $\mathrm{P}$ & 0.5 & WS & $F$ & 0.75 & UBI & $F$ & 0.75 \\
\hline UBI+WS & $\mathrm{F}$ & 0.75 & GMI+WS & $\mathrm{F}$ & 1 & $\mathrm{UBI}+\mathrm{WS}$ & $\mathrm{F}$ & 0.5 & UBI+WS & $\mathrm{F}$ & 0.75 & UBI+WS & $F$ & 0.75 & UBI & $\mathrm{P}$ & 0.75 \\
\hline WS & $\mathrm{F}$ & 0.75 & GMI & $\mathrm{P}$ & 0.5 & GMI+WS & $\mathrm{P}$ & 1 & GMI+WS & $\mathrm{F}$ & 1 & GMI+WS & $F$ & 1 & UBI+WS & $F$ & 0.75 \\
\hline GMI & $P$ & 0.5 & GMI & $\mathrm{P}$ & 1 & GMI+WS & $\mathrm{P}$ & 0.75 & WS & $\mathrm{F}$ & 0.5 & GMI+WS & $F$ & 0.75 & GMI & $\mathrm{P}$ & 0.75 \\
\hline GMI+WS & $\mathrm{F}$ & 1 & GMI & $\mathrm{P}$ & 0.75 & GMI+WS & $\mathrm{P}$ & 0.5 & GMI & $\mathrm{P}$ & 0.75 & WS & $\mathrm{F}$ & 0.5 & GMI & $\mathrm{F}$ & 0.75 \\
\hline GMI & $\mathrm{P}$ & 0.75 & CURRENT & & & GMI+WS & $\mathrm{F}$ & 0.5 & UBI+WS & $\mathrm{F}$ & 0.5 & GMI & $\mathrm{P}$ & 0.75 & WS & $F$ & 1 \\
\hline GMI & $\mathrm{P}$ & 1 & $\mathrm{UBI}+\mathrm{WS}$ & $F$ & 0.5 & $\mathrm{UBI}+\mathrm{WS}$ & P & 1 & GMI+WS & $\mathrm{F}$ & 0.75 & $\mathrm{UBI}+\mathrm{WS}$ & $F$ & 0.5 & GMI+WS & $F$ & 1 \\
\hline $\mathrm{CU}$ & & & GMI+WS & $F$ & 0.75 & WS & $\mathrm{F}$ & 1 & GMI+WS & $\mathrm{P}$ & 1 & UBI+WS & $P$ & 1 & WS & $\mathrm{P}$ & 1 \\
\hline UBI+WS & $F$ & 0.5 & GMI & $\mathrm{F}$ & 1 & WS & $\mathrm{P}$ & 1 & UBI+WS & $\mathrm{P}$ & 1 & GMI+WS & $P$ & 1 & UBI+WS & $\mathrm{P}$ & 1 \\
\hline GMI+WS & $F$ & 0.75 & GMI+WS & $F$ & 0.5 & WS & P & 0.5 & GMI+WS & $\mathrm{F}$ & 0.5 & GMI+WS & $F$ & 0.5 & GMI+WS & $\mathrm{P}$ & 1 \\
\hline WS & $F$ & 0.5 & GMI & $\mathrm{F}$ & 0.75 & WS & $F$ & 0.75 & CURRENT & & & GMI & $F$ & 0.75 & UBI & $F$ & 1 \\
\hline GMI+WS & $F$ & 0.5 & GMI & $\mathrm{F}$ & 0.5 & WS & $\mathrm{F}$ & 0.5 & GMI & $\mathrm{F}$ & 1 & CUR & EN? & & UBI+WS & $F$ & 1 \\
\hline GMI & $F$ & 1 & WS & $F$ & 1 & WS & P & 0.75 & GMI & $\mathrm{F}$ & 0.5 & GMI & $F$ & 1 & UBI & $\mathrm{P}$ & 1 \\
\hline GMI & $F$ & 0.75 & WS & $F$ & 0.75 & CUR & EN & & GMI & $F$ & 0.75 & GMI & $F$ & 0.5 & GMI & $\mathrm{P}$ & 1 \\
\hline GMI & $F$ & 0.5 & WS & $\mathrm{F}$ & 0.5 & UBI & I & 1 & GMI & $\mathrm{P}$ & 1 & GMI & $\mathrm{P}$ & 1 & GMI & $\mathrm{F}$ & 1 \\
\hline
\end{tabular}


Table 2. Policies ranked (from best to worst) according to the PAGSW criterion

\begin{tabular}{|c|c|c|c|c|c|c|c|c|c|c|c|c|c|c|c|c|c|}
\hline \multicolumn{3}{|c|}{ No behaviour } & \multicolumn{3}{|c|}{ No equilibrium } & \multicolumn{3}{|c|}{ Equilibrium $\eta=0$} & \multicolumn{3}{|c|}{ Equilibrium $\eta=-0.5$} & \multicolumn{3}{|c|}{ Equilibrium $\eta=-1$} & \multicolumn{3}{|c|}{ Equilibrium $\eta=$-oo } \\
\hline UBI & $P$ & 1 & UBI+WS & $\mathrm{P}$ & 1 & UBI & $\mathrm{F}$ & 1 & UBI & $\mathrm{P}$ & 1 & UBI & & 1 & UBI & & 0.75 \\
\hline UBI & F & 1 & UBI & $\mathrm{P}$ & 0.75 & UBI & $\mathrm{P}$ & 0.75 & UBI & F & 1 & UBI & F & 1 & UBI & $\mathrm{F}$ & 0.75 \\
\hline UBI & $\mathrm{F}$ & 0.75 & UBI & $\mathrm{P}$ & 1 & UBI & F & 0.75 & UBI & $P$ & 0.75 & UBI & $\mathrm{P}$ & 0.75 & UBI & $F$ & 1 \\
\hline GMI & $P$ & 1 & UBI & $\mathrm{P}$ & 0.5 & UBI & $\mathrm{P}$ & 1 & GMI & $\mathrm{F}$ & 1 & GMI & $F$ & 1 & UBI & $\mathrm{P}$ & 1 \\
\hline GMI & $\mathrm{F}$ & 1 & UBI+WS & $\mathrm{P}$ & 0.75 & GMI & $\mathrm{P}$ & 1 & GMI & $\mathrm{P}$ & 1 & GMI & P & 1 & GMI & $\mathrm{P}$ & 1 \\
\hline UBI & $\mathrm{F}$ & 0.75 & UBI & $\mathrm{F}$ & 1 & UBI+WS & $\mathrm{P}$ & 1 & UBI & F & 0.75 & UBI & F & 0.75 & GMI & $\mathrm{F}$ & 1 \\
\hline UBI+WS & $\mathrm{P}$ & 1 & UBI & $\mathrm{F}$ & 0.75 & GMI & F & 1 & UBI+WS & $P$ & 1 & UBI+WS & $\mathrm{P}$ & 1 & UBI & $\mathrm{P}$ & 0.5 \\
\hline UBI & $\mathrm{P}$ & 0.5 & UBI+WS & $\mathrm{P}$ & 0.5 & UBI & $\mathrm{P}$ & 0.5 & UBI & $P$ & 0.5 & UBI & $\mathrm{P}$ & 0.5 & UBI+WS & $\mathrm{P}$ & 1 \\
\hline UBI+WS & $\mathrm{F}$ & 1 & UBI+WS & $\mathrm{F}$ & 1 & UBI+WS & $\mathrm{F}$ & 1 & GMI & $\mathrm{P}$ & 0.75 & UBI+WS & F & 1 & GMI & $\mathrm{P}$ & 0.75 \\
\hline GMI & $\mathrm{P}$ & 0.75 & GMI & $\mathrm{P}$ & 1 & UBI+WS & $P$ & 0.75 & UBI+WS & $\mathrm{F}$ & 1 & GMI+WS & $\mathrm{P}$ & 1 & UBI & $\mathrm{F}$ & 0.5 \\
\hline UBI & $\mathrm{F}$ & 0.5 & UBI & $\mathrm{F}$ & 0.5 & GMI+WS & $\mathrm{P}$ & 1 & GMI+WS & $P$ & 1 & GMI & $P$ & 0.75 & GMI & $\mathrm{F}$ & 0.75 \\
\hline GMI & F & 0.75 & WS & $\mathrm{P}$ & 1 & UBI & $\mathrm{F}$ & 0.5 & UBI & F & 0.5 & UBI+WS & $P$ & 0.75 & UBI+WS & $\mathrm{P}$ & 0.75 \\
\hline UBI+WS & $\mathrm{P}$ & 0.75 & WS & $\mathrm{P}$ & 0.75 & GMI & $\mathrm{P}$ & 0.75 & UBI+WS & $P$ & 0.75 & UBI & $\mathrm{F}$ & 0.5 & UBI+WS & $F$ & 1 \\
\hline GMI+WS & $\mathrm{P}$ & 1 & WS & $\mathrm{P}$ & 0.5 & GMI & F & 0.75 & GMI & $F$ & 0.75 & GMI & $\mathrm{F}$ & 0.75 & GMI+WS & $\mathrm{P}$ & 1 \\
\hline GMI+WS & $\mathrm{F}$ & 1 & UBI+WS & $\mathrm{F}$ & 0.75 & GMI+WS & $\mathrm{F}$ & 1 & GMI+WS & F & 1 & GMI+WS & $\mathrm{F}$ & 1 & GMI+WS & $F$ & 1 \\
\hline UBI+WS & $\mathrm{F}$ & 0.75 & GMI & $\mathrm{P}$ & 0.75 & UBI+WS & $\mathrm{F}$ & 0.75 & UBI+WS & F & 0.75 & UBI+WS & $F$ & 0.75 & UBI+WS & $\mathrm{F}$ & 0.75 \\
\hline GMI & $\mathrm{P}$ & 0.5 & GMI+WS & $\mathrm{F}$ & 1 & GMI+WS & $\mathrm{P}$ & 0.75 & GMI+WS & $\mathrm{P}$ & 0.75 & GMI+WS & P & 0.75 & GMI+WS & $\mathrm{P}$ & 0.75 \\
\hline GMI+WS & $\mathrm{P}$ & 0.75 & GMI & $\mathrm{F}$ & 1 & UBI+WS & $P$ & 0.5 & GMI & $\mathrm{P}$ & 0.5 & GMI & $P$ & 0.5 & GMI & $\mathrm{P}$ & 0.5 \\
\hline UBI+WS & $\mathrm{P}$ & 0.5 & GMI+WS & $\mathrm{P}$ & 0.5 & GMI & $\mathrm{P}$ & 0.5 & UBI+WS & $\mathrm{P}$ & 0.5 & UBI+WS & $\mathrm{P}$ & 0.5 & UBI+WS & $\mathrm{P}$ & 0.5 \\
\hline GMI+WS & $\mathrm{F}$ & 0.75 & GMI & $\mathrm{P}$ & 0.5 & GMI+WS & $\mathrm{F}$ & 0.75 & GMI+WS & $\mathrm{F}$ & 0.75 & GMI+WS & F & 0.75 & GMI+WS & $\mathrm{F}$ & 0.75 \\
\hline GMI & F & 0.5 & GMI+WS & $\mathrm{P}$ & 0.75 & WS & P & 1 & WS & P & 1 & WS & $\mathrm{P}$ & 1 & GMI & $\mathrm{F}$ & 0.5 \\
\hline GMI+WS & $\mathrm{P}$ & 0.5 & current & & & GMI+WS & $\mathrm{P}$ & 0.5 & GMI & F & 0.5 & GMI & F & 0.5 & UBI+WS & $\mathrm{F}$ & 0.5 \\
\hline UBI+WS & $\mathrm{F}$ & 0.5 & GMI+WS & P & 1 & UBI+WS & $\mathrm{F}$ & 0.5 & UBI+WS & $F$ & 0.5 & UBI+WS & $F$ & 0.5 & WS & $\mathrm{P}$ & 1 \\
\hline WS & $\mathrm{P}$ & 1 & UBI+WS & $\mathrm{F}$ & 0.5 & GMI & $\mathrm{F}$ & 0.5 & GMI+WS & $P$ & 0.5 & GMI+WS & $P$ & 0.5 & GMI+WS & $\mathrm{P}$ & 0.5 \\
\hline WS & $\mathrm{F}$ & 1 & GMI+WS & $\mathrm{F}$ & 0.75 & WS & P & 0.75 & WS & $\mathrm{P}$ & 0.75 & WS & $\mathrm{P}$ & 0.75 & GMI+WS & $\mathrm{F}$ & 0.5 \\
\hline WS & $\mathrm{P}$ & 0.75 & GMI & $\mathrm{F}$ & 0.75 & WS & $\mathrm{F}$ & 1 & WS & F & 1 & WS & F & 1 & WS & $\mathrm{P}$ & 0.75 \\
\hline GMI+WS & $F$ & 0.5 & GMI+WS & $\mathrm{F}$ & 0.5 & GMI+WS & $\mathrm{F}$ & 0.5 & GMI+WS & $\mathrm{F}$ & 0.5 & GMI+WS & F & 0.5 & WS & $\mathrm{F}$ & 1 \\
\hline WS & $\mathrm{F}$ & 0.75 & GMI & $\mathrm{F}$ & 0.5 & WS & $F$ & 0.75 & WS & $F$ & 0.75 & WS & $F$ & 0.75 & & ENT & \\
\hline WS & $\mathrm{P}$ & 0.5 & WS & $\mathrm{F}$ & 1 & WS & P & 0.5 & WS & P & 0.5 & WS & $P$ & 0.5 & WS & $\mathrm{P}$ & 0.5 \\
\hline & ENT & & WS & $\mathrm{F}$ & 0.75 & & ENT & & & $\overline{\text { ENT }}$ & & $\mathrm{CU}$ & $\overline{\text { ENT }}$ & & WS & $\mathrm{F}$ & 0.75 \\
\hline WS & $\mathrm{F}$ & 0.5 & WS & $\mathrm{F}$ & 0.5 & WS & $F$ & 0.5 & WS & $F$ & 0.5 & WS & $F$ & 0.5 & WS & $\mathrm{F}$ & 0.5 \\
\hline
\end{tabular}


Table 3. Effects of policy attributes on Social Welfare. Regression coefficients (t-Statistics in parenthesis)

\begin{tabular}{|c|c|c|}
\hline & GSW & PAGSW \\
\hline Constant & $94233.08(12.22)$ & $88787.22(70.80)$ \\
\hline Progressive & $12.37(3.37)$ & $457.59(1.32)$ \\
\hline Coverage & $87.22(2.37)$ & $8260.96(2.19)$ \\
\hline Coverage $^{2}$ & $-65.46(-2.48)$ & $-2995.58(-1.11)$ \\
\hline Unconditional & $16.49(4.72)$ & $274.49(0.77)$ \\
\hline Subsidy & $2.16(0.62)$ & $-1944.72(-5.43)$ \\
\hline
\end{tabular}

Note to Table 3

Progressive $=1$ if tax rule is progressive $(0$ otherwise $)$

Coverage $=$ the value of $a$ (for the CURRENT system we set $a=0.1$ );

Coverage $^{2}=$ Coverage squared;

Unconditional $=1$ if income support mechanism is UBI or UBI+WS ( 0 otherwise);

Subsidy $=1$ if income support mechanism is WS or UBI+WS or GMI+WS (0 otherwise). 


\section{Table 4. Legenda for Tables 5 - 10}

(a) = either the CURRENT tax-transfer rule or a reform: the first label refers to the income support mechanism, the second label denotes flat $(\mathrm{F})$ or progressive $(\mathrm{P})$ marginal tax rates, the last number is the guaranteed minimum income as a proportion of the poverty line.

(b): average male weekly expected hours of work (including the 0 hours of non participants).

(c): average female weekly expected hours of work (including the 0 hours of non participants).

(d): average monthly gross income (Euros 1998).

(e): average monthly net available income (Euros 1998).

(f): for Flat tax rules, it is the constant tax rates; for Progressive tax rules, it is the proportional increase with respect to the current marginal rates (reported in Appendix C).

(g): average monthly transfer (Euros 1998).

(h): proportion of utility-winners (household $n$ is a utility-winner under reform $R_{1}$ with respect to the current system $R_{0}$ if $\mu^{n}\left(R_{1}\right)>\mu^{n}\left(R_{0}\right)$ - see section 4$)$.

(i): proportion of income-winners (a household is a income-winner if household's net available income is higher under the reform than under the current system).

(1): poverty ratio (head-count rate) $=$ number of poor as a percentage of the number of households in the sample.

(m): poverty-gap ratio = average distance between the poverty line and the incomes of the poor, as a percentage of the poverty line.

(n): income-gap ratio = distance between the poverty line and the average income of the poor, as a percentage of the poverty line.

(o): Gini Social Welfare.

(p): Poverty-adjusted Gini Social Welfare. 
Table 5. No Behaviour

\begin{tabular}{|c|c|c|c|c|c|c|c|c|c|c|c|c|c|c|c|}
\hline \multicolumn{3}{|c|}{ a) } & (b) & (c) & (d) & (e) & (f) & $(\mathrm{g})$ & (h) & (i) & (l) & $(\mathrm{m})$ & (n) & (o) & (p) \\
\hline \multicolumn{3}{|c|}{ 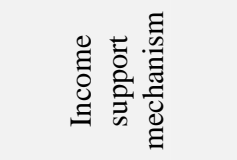 } & 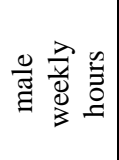 & 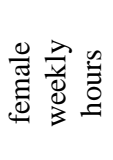 & 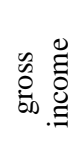 & 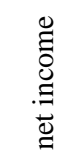 & 离 & 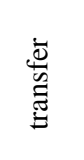 & 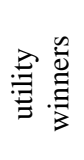 & 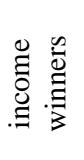 & 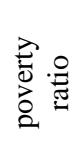 & 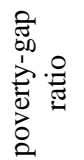 & 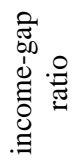 & 勇 & $\begin{array}{l}3 \\
0 \\
\vdots \\
0 \\
2\end{array}$ \\
\hline \multicolumn{3}{|c|}{ CURRENT } & 39.35 & 19.45 & 2930 & 2191 & -- & 101 & -- & -- & 4.23 & 0.58 & 13.71 & 81466 & 77829 \\
\hline GMI & $\mathrm{F}$ & 0.5 & 39.35 & 19.45 & 2930 & 2194 & 0.31 & 192 & 0.38 & 0.57 & 2.95 & 0.31 & 10.51 & 81449 & 78911 \\
\hline GMI & $F$ & 0.75 & 39.35 & 19.45 & 2930 & 2196 & 0.37 & 265 & 0.37 & 0.60 & 0.97 & 0.06 & 6.50 & 81451 & 80619 \\
\hline GMI & $F$ & 1 & 39.35 & 19.45 & 2930 & 2201 & 0.45 & 349 & 0.41 & 0.58 & 0.01 & 0.00 & 0.81 & 81452 & 81447 \\
\hline GMI & $\mathrm{P}$ & 0.5 & 39.34 & 19.45 & 2930 & 2191 & 0.02 & 192 & 0.52 & 0.63 & 2.38 & 0.25 & 10.63 & 81469 & 79427 \\
\hline GMI & $\mathrm{P}$ & 0.75 & 39.34 & 19.45 & 2930 & 2192 & 0.07 & 265 & 0.49 & 0.60 & 0.87 & 0.06 & 6.69 & 81468 & 80723 \\
\hline GMI & $\mathrm{P}$ & 1 & 39.33 & 19.45 & 2930 & 2195 & 0.13 & 349 & 0.47 & 0.60 & 0.02 & 0.00 & 1.12 & 81467 & 81448 \\
\hline GMI+WS & $F$ & 0.5 & 39.35 & 19.45 & 2930 & 2197 & 0.36 & 405 & 0.46 & 0.58 & 3.82 & 0.50 & 13.06 & 81457 & 78175 \\
\hline GMI+WS & $\mathrm{F}$ & 0.75 & 39.35 & 19.45 & 2930 & 2198 & 0.40 & 435 & 0.48 & 0.62 & 2.65 & 0.28 & 10.49 & 81463 & 79189 \\
\hline GMI+WS & $\mathrm{F}$ & 1 & 39.35 & 19.45 & 2930 & 2200 & 0.45 & 467 & 0.51 & 0.64 & 1.53 & 0.12 & 8.11 & 81469 & 80151 \\
\hline GMI+WS & $\mathrm{P}$ & 0.5 & 39.35 & 19.45 & 2930 & 2187 & 0.06 & 405 & 0.61 & 0.63 & 3.25 & 0.41 & 12.63 & 81478 & 78686 \\
\hline GMI+WS & $\mathrm{P}$ & 0.75 & 39.35 & 19.45 & 2930 & 2183 & 0.09 & 435 & 0.59 & 0.61 & 2.40 & 0.26 & 10.65 & 81478 & 79418 \\
\hline GMI+WS & $\mathrm{P}$ & 1 & 39.35 & 19.45 & 2930 & 2179 & 0.13 & 467 & 0.57 & 0.59 & 1.46 & 0.13 & 9.15 & 81479 & 80226 \\
\hline UBI & $\mathrm{F}$ & 0.5 & 39.35 & 19.45 & 2930 & 2205 & 0.41 & 568 & 0.53 & 0.63 & 0.91 & 0.06 & 6.95 & 81474 & 80693 \\
\hline UBI & $\mathrm{F}$ & 0.75 & 39.35 & 19.45 & 2930 & 2210 & 0.50 & 814 & 0.53 & 0.60 & 0.08 & 0.00 & 2.80 & 81480 & 81413 \\
\hline UBI & $\mathrm{F}$ & 1 & 39.35 & 19.45 & 2930 & 2214 & 0.60 & 1060 & 0.53 & 0.60 & 0.00 & 0.00 & 0.00 & 81480 & 81480 \\
\hline UBI & $\mathrm{P}$ & 0.5 & 39.35 & 19.45 & 2930 & 2204 & 0.13 & 568 & 0.62 & 0.63 & 0.60 & 0.04 & 6.34 & 81493 & 80979 \\
\hline UBI & $\mathrm{P}$ & 0.75 & 39.35 & 19.45 & 2930 & 2209 & 0.22 & 814 & 0.59 & 0.63 & 0.04 & 0.00 & 3.36 & 81494 & 81464 \\
\hline UBI & $\mathrm{P}$ & 1 & 39.35 & 19.45 & 2930 & 2213 & 0.32 & 1060 & 0.56 & 0.62 & 0.00 & 0.00 & 0.00 & 81490 & 81490 \\
\hline UBI+WS & $\mathrm{F}$ & 0.5 & 39.35 & 19.45 & 2930 & 2200 & 0.38 & 598 & 0.50 & 0.61 & 3.29 & 0.38 & 11.64 & 81463 & 78631 \\
\hline UBI+WS & $\mathrm{F}$ & 0.75 & 39.35 & 19.45 & 2930 & 2203 & 0.42 & 721 & 0.53 & 0.64 & 1.96 & 0.17 & 8.84 & 81469 & 79786 \\
\hline UBI+WS & $\mathrm{F}$ & 1 & 39.35 & 19.45 & 2930 & 2206 & 0.47 & 844 & 0.53 & 0.63 & 0.83 & 0.05 & 5.92 & 81475 & 80761 \\
\hline $\mathrm{UBI}+\mathrm{WS}$ & $\mathrm{P}$ & 0.5 & 39.35 & 19.45 & 2930 & 2201 & 0.10 & 598 & 0.68 & 0.67 & 2.46 & 0.24 & 9.88 & 81489 & 79373 \\
\hline UBI+WS & $\mathrm{P}$ & 0.75 & 39.35 & 19.45 & 2930 & 2205 & 0.14 & 721 & 0.66 & 0.65 & 1.15 & 0.08 & 7.08 & 81494 & 80505 \\
\hline UBI+WS & $\mathrm{P}$ & 1 & 39.35 & 19.45 & 2930 & 2210 & 0.18 & 844 & 0.64 & 0.64 & 0.33 & 0.01 & 4.40 & 81501 & 81218 \\
\hline WS & $\mathrm{F}$ & 0.5 & 39.35 & 19.45 & 2930 & 2201 & 0.34 & 352 & 0.51 & 0.57 & 4.59 & 0.75 & 16.29 & 81462 & 77518 \\
\hline WS & $\mathrm{F}$ & 0.75 & 39.35 & 19.45 & 2930 & 2203 & 0.36 & 352 & 0.57 & 0.62 & 4.05 & 0.60 & 14.92 & 81469 & 77988 \\
\hline WS & $\mathrm{F}$ & 1 & 39.35 & 19.45 & 2930 & 2205 & 0.39 & 352 & 0.62 & 0.64 & 3.55 & 0.49 & 13.86 & 81477 & 78420 \\
\hline WS & $\mathrm{P}$ & 0.5 & 39.35 & 19.45 & 2930 & 2191 & 0.04 & 352 & 0.65 & 0.64 & 4.12 & 0.63 & 15.30 & 81483 & 77939 \\
\hline WS & $\mathrm{P}$ & 0.75 & 39.35 & 19.45 & 2930 & 2189 & 0.05 & 352 & 0.67 & 0.64 & 3.69 & 0.55 & 14.98 & 81487 & 78312 \\
\hline WS & $\mathrm{P}$ & 1 & 39.35 & 19.45 & 2930 & 2186 & 0.07 & 352 & 0.69 & 0.64 & 3.39 & 0.48 & 14.29 & 81491 & 78574 \\
\hline
\end{tabular}




\begin{tabular}{|c|c|c|c|c|c|c|c|c|c|c|c|c|c|c|c|}
\hline \multicolumn{16}{|c|}{ Table 6. No Equilibrium } \\
\hline \multicolumn{3}{|l|}{ a) } & (b) & (c) & (d) & (e) & (f) & (g) & (h) & (i) & (l) & $(\mathrm{m})$ & (n) & (o) & (p) \\
\hline \multicolumn{3}{|c|}{ 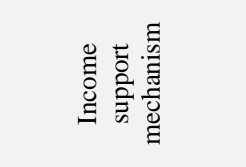 } & 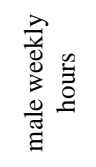 & 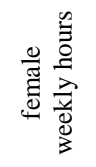 & 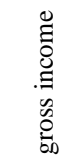 & 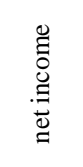 & 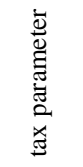 & 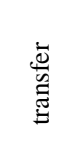 & 莺泀 & 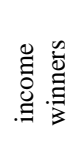 & 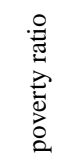 & 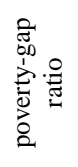 & 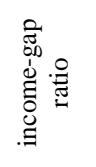 & 3 & 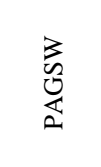 \\
\hline \multicolumn{3}{|c|}{ CURRENT } & 39.35 & 19.45 & 2930 & 2191 & -- & 101 & -- & -- & 4.23 & 0.58 & 13.71 & 94255 & 86391 \\
\hline GMI & $\mathrm{F}$ & 0.5 & 39.32 & 19.22 & 2925 & 2190 & 0.31 & 194 & 0.39 & 0.56 & 2.97 & 0.31 & 10.58 & 94241 & 86375 \\
\hline GMI & $\mathrm{F}$ & 0.75 & 39.28 & 19.07 & 2912 & 2183 & 0.38 & 269 & 0.37 & 0.55 & 0.93 & 0.06 & 6.39 & 94244 & 863835 \\
\hline GMI & $\mathrm{F}$ & 1 & 39.23 & 18.91 & 2896 & 2176 & 0.46 & 357 & 0.40 & 0.53 & 0.00 & 0.00 & 0.16 & 94248 & 86394 \\
\hline GMI & $\mathrm{P}$ & 0.5 & 39.31 & 19.24 & 2922 & 2185 & 0.02 & 194 & 0.52 & 0.62 & 2.36 & 0.25 & 10.55 & 94259 & 86393 \\
\hline GMI & $\mathrm{P}$ & 0.75 & 39.27 & 19.08 & 2909 & 2176 & 0.07 & 269 & 0.47 & 0.57 & 0.87 & 0.05 & 6.22 & 94259 & 86397 \\
\hline GMI & $\mathrm{P}$ & 1 & 39.22 & 18.91 & 2892 & 2168 & 0.14 & 357 & 0.45 & 0.55 & 0.01 & 0.00 & 0.66 & 94259 & 86404 \\
\hline GMI+WS & $\mathrm{F}$ & 0.5 & 39.34 & 19.38 & 2932 & 2198 & 0.36 & 406 & 0.47 & 0.59 & 3.90 & 0.51 & 13.11 & 94248 & 86380 \\
\hline GMI+WS & $\mathrm{F}$ & 0.75 & 39.32 & 19.31 & 2926 & 2195 & 0.40 & 435 & 0.49 & 0.61 & 2.69 & 0.28 & 10.40 & 94253 & 86387 \\
\hline GMI+WS & $\mathrm{F}$ & 1 & 39.30 & 19.24 & 2919 & 2191 & 0.45 & 467 & 0.50 & 0.60 & 1.38 & 0.12 & 8.68 & 94260 & 86394 \\
\hline GMI+WS & $\mathrm{P}$ & 0.5 & 39.33 & 19.39 & 2930 & 2187 & 0.06 & 405 & 0.60 & 0.63 & 3.26 & 0.41 & 12.63 & 94267 & 86393 \\
\hline GMI+WS & $\mathrm{P}$ & 0.75 & 39.31 & 19.32 & 2923 & 2177 & 0.09 & 435 & 0.58 & 0.59 & 2.34 & 0.25 & 10.64 & 94268 & 86392 \\
\hline GMI+WS & $\mathrm{P}$ & 1 & 39.29 & 19.24 & 2915 & 2167 & 0.13 & 467 & 0.56 & 0.56 & 1.43 & 0.13 & 8.96 & 94270 & 86391 \\
\hline UBI & $\mathrm{F}$ & 0.5 & 39.28 & 19.22 & 2915 & 2192 & 0.41 & 568 & 0.51 & 0.59 & 0.86 & 0.06 & 6.84 & 94265 & 86404 \\
\hline UBI & $\mathrm{F}$ & 0.75 & 39.23 & 19.06 & 2897 & 2183 & 0.51 & 814 & 0.51 & 0.56 & 0.06 & 0.00 & 2.91 & 94271 & 86415 \\
\hline UBI & $\mathrm{F}$ & 1 & 39.17 & 18.90 & 2876 & 2172 & 0.61 & 1060 & 0.50 & 0.55 & 0.00 & 0.00 & 0.00 & 94272 & 86420 \\
\hline UBI & $\mathrm{P}$ & 0.5 & 39.27 & 19.23 & 2907 & 2185 & 0.13 & 568 & 0.60 & 0.60 & 0.52 & 0.04 & 6.73 & 94283 & 86422 \\
\hline UBI & $\mathrm{P}$ & 0.75 & 39.21 & 19.06 & 2885 & 2173 & 0.23 & 814 & 0.56 & 0.58 & 0.04 & 0.00 & 2.52 & 94285 & 86428 \\
\hline UBI & $\mathrm{P}$ & 1 & 39.15 & 18.89 & 2859 & 2158 & 0.33 & 1060 & 0.54 & 0.57 & 0.00 & 0.00 & 0.00 & 94280 & 86428 \\
\hline UBI+WS & $\mathrm{F}$ & 0.5 & 39.33 & 19.36 & 2930 & 2200 & 0.38 & 598 & 0.51 & 0.61 & 3.30 & 0.40 & 12.04 & 94253 & 86389 \\
\hline $\mathrm{UBI}+\mathrm{WS}$ & $\mathrm{F}$ & 0.75 & 39.31 & 19.28 & 2922 & 2196 & 0.43 & 720 & 0.52 & 0.62 & 1.96 & 0.17 & 8.78 & 94260 & 86398 \\
\hline UBI+WS & $\mathrm{F}$ & 1 & 39.28 & 19.21 & 2914 & 2193 & 0.47 & 843 & 0.51 & 0.60 & 0.73 & 0.05 & 6.25 & 94266 & 86407 \\
\hline UBI+WS & $\mathrm{P}$ & 0.5 & 39.32 & 19.38 & 2924 & 2196 & 0.10 & 597 & 0.66 & 0.66 & 2.44 & 0.24 & 9.75 & 94279 & 86414 \\
\hline UBI+WS & $\mathrm{P}$ & 0.75 & 39.29 & 19.30 & 2914 & 2193 & 0.14 & 719 & 0.64 & 0.62 & 1.01 & 0.07 & 7.43 & 94285 & 86422 \\
\hline UBI+WS & $\mathrm{P}$ & 1 & 39.26 & 19.22 & 2903 & 2189 & 0.19 & 842 & 0.61 & 0.61 & 0.17 & 0.01 & 6.80 & 94292 & 86430 \\
\hline WS & $\mathrm{F}$ & 0.5 & 39.36 & 19.49 & 2921 & 2205 & 0.34 & 352 & 0.52 & 0.58 & 4.67 & 0.76 & 16.29 & 91925 & 85178 \\
\hline WS & $\mathrm{F}$ & 0.75 & 39.36 & 19.50 & 2919 & 2207 & 0.36 & 352 & 0.57 & 0.63 & 4.16 & 0.62 & 14.87 & 91933 & 85186 \\
\hline WS & $\mathrm{F}$ & 1 & 39.35 & 19.50 & 2918 & 2208 & 0.39 & 352 & 0.62 & 0.65 & 3.56 & 0.50 & 13.91 & 91941 & 85194 \\
\hline WS & $\mathrm{P}$ & 0.5 & 39.36 & 19.53 & 2922 & 2197 & 0.04 & 352 & 0.65 & 0.65 & 4.11 & 0.64 & 15.48 & 94272 & 86400 \\
\hline WS & $\mathrm{P}$ & 0.75 & 39.35 & 19.52 & 2920 & 2193 & 0.05 & 352 & 0.68 & 0.65 & 3.67 & 0.55 & 15.01 & 94276 & 86401 \\
\hline WS & $\mathrm{P}$ & 1 & 39.35 & 19.53 & 2918 & 2189 & 0.07 & 352 & 0.69 & 0.64 & 3.38 & 0.48 & 14.21 & 94280 & 86402 \\
\hline
\end{tabular}




\begin{tabular}{|c|c|c|c|c|c|c|c|c|c|c|c|c|c|c|c|}
\hline \multicolumn{16}{|c|}{ Table 7. Equilibrium: $\eta=0$} \\
\hline \multicolumn{3}{|c|}{ (a) } & (b) & (c) & (d) & (e) & (f) & (g) & (h) & (i) & (l) & $(\mathrm{m})$ & (n) & (o) & (p) \\
\hline \multicolumn{3}{|c|}{ 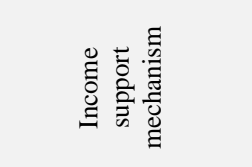 } & 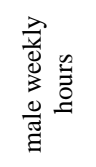 & 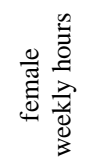 & 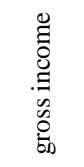 & 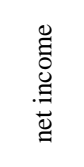 & 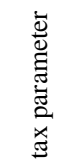 & 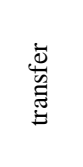 & 常高 & 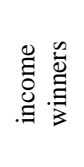 & 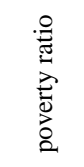 & 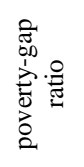 & 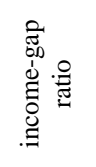 & 莕 & $\begin{array}{l}3 \\
0 \\
0 \\
\vdots \\
0\end{array}$ \\
\hline \multicolumn{3}{|c|}{ CURRENT } & 39.35 & 19.45 & 2930 & 2191 & -- & 101 & -- & -- & 4.23 & 0.58 & 13.71 & 94255 & 90047 \\
\hline GMI & $\mathrm{F}$ & 0.5 & 39.39 & 19.50 & 3193 & 2433 & 0.29 & 191 & 0.83 & 0.91 & 3.60 & 0.40 & 10.98 & 94324 & 90745 \\
\hline GMI & $\mathrm{F}$ & 0.75 & 39.38 & 19.47 & 3306 & 2533 & 0.33 & 260 & 0.88 & 0.95 & 1.81 & 0.15 & 8.13 & 94363 & 92561 \\
\hline GMI & $\mathrm{F}$ & 1 & 39.36 & 19.38 & 3360 & 2587 & 0.39 & 339 & 0.89 & 0.95 & 0.58 & 0.02 & 4.05 & 94388 & 93809 \\
\hline GMI & $\mathrm{P}$ & 0.5 & 39.36 & 19.45 & 3117 & 2366 & 0.00 & 191 & 0.84 & 0.89 & 2.94 & 0.31 & 10.50 & 94326 & 91399 \\
\hline GMI & $\mathrm{P}$ & 0.75 & 39.35 & 19.36 & 3174 & 2421 & 0.02 & 263 & 0.87 & 0.92 & 1.30 & 0.10 & 7.38 & 94345 & 93052 \\
\hline GMI & $\mathrm{P}$ & 1 & 39.31 & 19.26 & 3234 & 2476 & 0.07 & 342 & 0.86 & 0.91 & 0.21 & 0.01 & 4.61 & 94352 & 94148 \\
\hline GMI+WS & $\mathrm{F}$ & 0.5 & 39.37 & 19.50 & 3043 & 2307 & 0.35 & 406 & 0.72 & 0.80 & 4.04 & 0.54 & 13.42 & 94285 & 90266 \\
\hline GMI+WS & $\mathrm{F}$ & 0.75 & 39.37 & 19.48 & 3086 & 2346 & 0.38 & 435 & 0.80 & 0.87 & 3.00 & 0.33 & 10.88 & 94306 & 91321 \\
\hline GMI+WS & $\mathrm{F}$ & 1 & 39.35 & 19.44 & 3114 & 2372 & 0.42 & 466 & 0.82 & 0.89 & 1.85 & 0.17 & 9.10 & 94322 & 92477 \\
\hline GMI+WS & $\mathrm{P}$ & 0.5 & 39.35 & 19.46 & 2976 & 2246 & 0.05 & 405 & 0.74 & 0.76 & 3.44 & 0.44 & 12.71 & 94289 & 90871 \\
\hline GMI+WS & $\mathrm{P}$ & 0.75 & 39.33 & 19.39 & 2969 & 2245 & 0.07 & 435 & 0.74 & 0.77 & 2.42 & 0.25 & 10.10 & 94290 & 91879 \\
\hline GMI+WS & $\mathrm{P}$ & 1 & 39.31 & 19.37 & 3041 & 2306 & 0.10 & 466 & 0.78 & 0.80 & 1.12 & 0.11 & 9.59 & 94299 & 93182 \\
\hline UBI & $\mathrm{F}$ & 0.5 & 39.35 & 19.48 & 3170 & 2426 & 0.38 & 568 & 0.86 & 0.92 & 1.28 & 0.10 & 7.43 & 94348 & 93079 \\
\hline UBI & $\mathrm{F}$ & 0.75 & 39.31 & 19.35 & 3166 & 2430 & 0.47 & 814 & 0.84 & 0.89 & 0.12 & 0.01 & 6.13 & 94361 & 94238 \\
\hline UBI & $\mathrm{F}$ & 1 & 39.23 & 19.07 & 3027 & 2317 & 0.58 & 1060 & 0.69 & 0.71 & 0.00 & 0.00 & 0.00 & 94328 & 94328 \\
\hline UBI & $\mathrm{P}$ & 0.5 & 39.31 & 19.40 & 3064 & 2334 & 0.11 & 568 & 0.83 & 0.83 & 0.62 & 0.05 & 7.97 & 94340 & 93727 \\
\hline UBI & $\mathrm{P}$ & 0.75 & 39.23 & 19.15 & 2951 & 2244 & 0.22 & 814 & 0.67 & 0.67 & 0.06 & 0.00 & 2.94 & 94313 & 94258 \\
\hline UBI & $\mathrm{P}$ & 1 & 39.08 & 18.67 & 2646 & 1986 & 0.38 & 1060 & 0.38 & 0.44 & 0.00 & 0.00 & 0.00 & 94206 & 94206 \\
\hline UBI+WS & $\mathrm{F}$ & 0.5 & 39.37 & 19.51 & 3069 & 2333 & 0.37 & 599 & 0.79 & 0.85 & 3.49 & 0.43 & 12.25 & 94300 & 90830 \\
\hline UBI+WS & $\mathrm{F}$ & 0.75 & 39.36 & 19.48 & 3113 & 2374 & 0.41 & 721 & 0.83 & 0.89 & 2.21 & 0.21 & 9.65 & 94323 & 92128 \\
\hline UBI+WS & $F$ & 1 & 39.34 & 19.43 & 3131 & 2394 & 0.44 & 844 & 0.84 & 0.90 & 1.02 & 0.08 & 7.66 & 94337 & 93322 \\
\hline UBI+WS & $\mathrm{P}$ & 0.5 & 39.35 & 19.43 & 2988 & 2264 & 0.09 & 598 & 0.78 & 0.79 & 2.58 & 0.27 & 10.46 & 94303 & 91732 \\
\hline UBI+WS & $\mathrm{P}$ & 0.75 & 39.31 & 19.34 & 2960 & 2248 & 0.11 & 720 & 0.74 & 0.73 & 1.00 & 0.08 & 7.62 & 94301 & 93311 \\
\hline UBI+WS & $\mathrm{P}$ & 1 & 39.26 & 19.24 & 2964 & 2249 & 0.16 & 841 & 0.66 & 0.64 & 0.27 & 0.01 & 3.56 & 94281 & 94013 \\
\hline WS & $\mathrm{F}$ & 0.5 & 39.37 & 19.53 & 2944 & 2225 & 0.34 & 353 & 0.57 & 0.63 & 4.67 & 0.76 & 16.33 & 94258 & 89610 \\
\hline WS & $\mathrm{F}$ & 0.75 & 39.36 & 19.53 & 2940 & 2224 & 0.36 & 352 & 0.62 & 0.66 & 4.17 & 0.62 & 14.88 & 94265 & 90120 \\
\hline WS & $\mathrm{F}$ & 1 & 39.36 & 19.53 & 2933 & 2221 & 0.39 & 352 & 0.65 & 0.68 & 3.65 & 0.50 & 13.79 & 94271 & 90646 \\
\hline WS & $\mathrm{P}$ & 0.5 & 39.35 & 19.50 & 2895 & 2180 & 0.04 & 352 & 0.63 & 0.62 & 4.18 & 0.64 & 15.19 & 94266 & 90106 \\
\hline WS & $\mathrm{P}$ & 0.75 & 39.34 & 19.47 & 2857 & 2153 & 0.04 & 352 & 0.57 & 0.54 & 3.63 & 0.52 & 14.45 & 94258 & 90649 \\
\hline WS & $\mathrm{P}$ & 1 & 39.34 & 19.52 & 2920 & 2206 & 0.07 & 351 & 0.67 & 0.65 & 3.01 & 0.38 & 12.46 & 94268 & 91273 \\
\hline
\end{tabular}


Table 8. Equilibrium: $\eta=\mathbf{- 0 . 5}$

\begin{tabular}{|c|c|c|c|c|c|c|c|c|c|c|c|c|c|c|c|}
\hline \multicolumn{3}{|c|}{ (a) } & (b) & (c) & (d) & (e) & (f) & (g) & (h) & (i) & (1) & $(\mathrm{m})$ & (n) & (o) & (p) \\
\hline \multicolumn{3}{|c|}{ 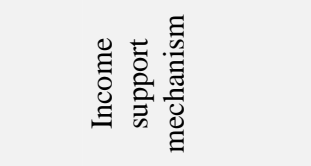 } & 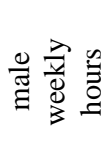 & 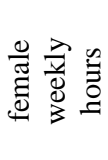 & 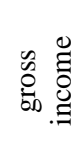 & 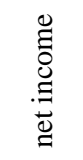 & 离 & 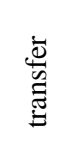 & 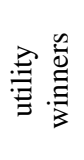 & 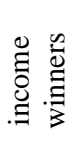 & 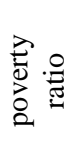 & 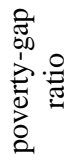 & 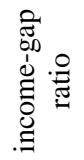 & 3 & $\begin{array}{l}3 \\
0 \\
\vdots \\
\vdots \\
2\end{array}$ \\
\hline \multicolumn{3}{|c|}{ CURRENT } & 39.35 & 19.45 & 2930 & 2191 & -- & 101 & -- & -- & 4.23 & 0.58 & 13.71 & 94255 & 90047 \\
\hline GMI & $\mathrm{F}$ & 0.5 & 39.31 & 19.27 & 2923 & 2202 & 0.31 & 193 & 0.46 & 0.60 & 3.09 & 0.32 & 10.37 & 94248 & 91176 \\
\hline GMI & $\mathrm{F}$ & 0.75 & 39.26 & 19.10 & 2906 & 2191 & 0.38 & 269 & 0.42 & 0.58 & 0.92 & 0.06 & 0.61 & 94247 & 93333 \\
\hline GMI & $\mathrm{F}$ & 1 & 39.21 & 18.94 & 2889 & 2183 & 0.45 & 356 & 0.41 & 0.54 & 0.00 & 0.00 & 0.18 & 94251 & 94248 \\
\hline GMI & $\mathrm{P}$ & 0.5 & 39.30 & 19.29 & 2921 & 2198 & 0.02 & 193 & 0.59 & 0.65 & 2.50 & 0.26 & 10.47 & 94269 & 91785 \\
\hline GMI & $\mathrm{P}$ & 0.75 & 39.26 & 19.12 & 2903 & 2189 & 0.05 & 269 & 0.52 & 0.59 & 0.65 & 0.05 & 7.41 & 94262 & 93613 \\
\hline GMI & $\mathrm{P}$ & 1 & 39.19 & 18.93 & 2877 & 2169 & 0.14 & 356 & 0.43 & 0.52 & 0.01 & 0.00 & 0.23 & 94242 & 94237 \\
\hline GMI+WS & $\mathrm{F}$ & 0.5 & 39.33 & 19.42 & 2932 & 2211 & 0.36 & 406 & 0.52 & 0.62 & 3.88 & 0.51 & 13.19 & 94256 & 90398 \\
\hline GMI+WS & $\mathrm{F}$ & 0.75 & 39.31 & 19.34 & 2923 & 2206 & 0.40 & 435 & 0.54 & 0.64 & 2.77 & 0.28 & 10.24 & 94260 & 91511 \\
\hline GMI+WS & $\mathrm{F}$ & 1 & 39.29 & 19.28 & 2916 & 2202 & 0.45 & 467 & 0.56 & 0.64 & 1.54 & 0.12 & 8.07 & 94266 & 92732 \\
\hline GMI+WS & $\mathrm{P}$ & 0.5 & 38.76 & 20.13 & 2937 & 2212 & 0.06 & 408 & 0.63 & 0.70 & 3.43 & 0.42 & 12.29 & 94279 & 90864 \\
\hline GMI+WS & $\mathrm{P}$ & 0.75 & 38.72 & 20.08 & 2929 & 2210 & 0.07 & 437 & 0.63 & 0.68 & 2.36 & 0.24 & 10.05 & 94278 & 91928 \\
\hline GMI+WS & $\mathrm{P}$ & 1 & 38.66 & 20.00 & 2912 & 2193 & 0.13 & 468 & 0.54 & 0.57 & 0.81 & 0.09 & 11.17 & 94258 & 93455 \\
\hline UBI & $\mathrm{F}$ & 0.5 & 39.27 & 19.26 & 2910 & 2202 & 0.41 & 568 & 0.56 & 0.62 & 0.87 & 0.06 & 7.08 & 94270 & 93410 \\
\hline UBI & $\mathrm{F}$ & 0.75 & 39.22 & 19.09 & 2889 & 2190 & 0.51 & 814 & 0.52 & 0.57 & 0.06 & 0.00 & 3.11 & 94272 & 94215 \\
\hline UBI & $\mathrm{F}$ & 1 & 39.16 & 18.93 & 2867 & 2178 & 0.60 & 1060 & 0.51 & 0.56 & 0.00 & 0.00 & 0.00 & 94273 & 94273 \\
\hline UBI & $\mathrm{P}$ & 0.5 & 39.26 & 19.26 & 2902 & 2195 & 0.13 & 568 & 0.62 & 0.62 & 0.52 & 0.04 & 6.96 & 94289 & 93770 \\
\hline UBI & $\mathrm{P}$ & 0.75 & 39.20 & 19.09 & 2877 & 2180 & 0.23 & 814 & 0.57 & 0.59 & 0.04 & 0.00 & 2.78 & 94287 & 94252 \\
\hline UBI & $\mathrm{P}$ & 1 & 39.13 & 18.92 & 2850 & 2164 & 0.33 & 1060 & 0.53 & 0.58 & 0.00 & 0.00 & 0.00 & 94281 & 94281 \\
\hline UBI+WS & $\mathrm{F}$ & 0.5 & 39.32 & 19.40 & 2928 & 2212 & 0.38 & 598 & 0.56 & 0.65 & 3.32 & 0.40 & 12.07 & 94262 & 90961 \\
\hline UBI+WS & $\mathrm{F}$ & 0.75 & 39.30 & 19.33 & 2920 & 2208 & 0.42 & 720 & 0.57 & 0.65 & 1.96 & 0.18 & 8.90 & 94268 & 92316 \\
\hline UBI+WS & $\mathrm{F}$ & 1 & 39.27 & 19.24 & 2910 & 2203 & 0.47 & 843 & 0.56 & 0.62 & 0.73 & 0.05 & 6.38 & 94272 & 93545 \\
\hline UBI+WS & $\mathrm{P}$ & 0.5 & 39.31 & 19.42 & 2924 & 2208 & 0.09 & 598 & 0.68 & 0.69 & 2.52 & 0.26 & 10.14 & 94288 & 91778 \\
\hline UBI+WS & $\mathrm{P}$ & 0.75 & 39.27 & 19.34 & 2911 & 2205 & 0.12 & 720 & 0.64 & 0.64 & 0.89 & 0.07 & 7.70 & 94290 & 93405 \\
\hline UBI+WS & $\mathrm{P}$ & 1 & 39.21 & 19.21 & 2886 & 2180 & 0.18 & 841 & 0.51 & 0.53 & 0.23 & 0.01 & 2.61 & 94257 & 94029 \\
\hline WS & $\mathrm{F}$ & 0.5 & 39.36 & 19.55 & 2940 & 2222 & 0.34 & 353 & 0.55 & 0.62 & 4.64 & 0.76 & 16.43 & 94262 & 89650 \\
\hline WS & $\mathrm{F}$ & 0.75 & 39.35 & 19.55 & 2938 & 2223 & 0.36 & 352 & 0.59 & 0.66 & 4.16 & 0.62 & 14.95 & 94270 & 90137 \\
\hline WS & $\mathrm{F}$ & 1 & 39.34 & 19.56 & 2936 & 2223 & 0.39 & 352 & 0.62 & 0.68 & 3.65 & 0.50 & 13.79 & 94277 & 90651 \\
\hline WS & $\mathrm{P}$ & 0.5 & 39.35 & 19.57 & 2938 & 2218 & 0.03 & 352 & 0.66 & 0.71 & 4.18 & 0.65 & 15.57 & 94285 & 90131 \\
\hline WS & $\mathrm{P}$ & 0.75 & 39.35 & 19.57 & 2937 & 2222 & 0.03 & 352 & 0.68 & 0.73 & 3.63 & 0.54 & 14.82 & 94289 & 90677 \\
\hline WS & $\mathrm{P}$ & 1 & 39.33 & 19.55 & 2928 & 2213 & 0.06 & 352 & 0.64 & 0.66 & 3.01 & 0.38 & 12.59 & 94275 & 91280 \\
\hline
\end{tabular}




\begin{tabular}{|c|c|c|c|c|c|c|c|c|c|c|c|c|c|c|c|}
\hline \multicolumn{16}{|c|}{ Table 9. Equilibrium: $\eta=-1$} \\
\hline \multicolumn{3}{|c|}{ (a) } & (b) & (c) & (d) & (e) & (f) & (g) & (h) & (i) & (1) & (m) & (n) & (o) & (p) \\
\hline \multicolumn{3}{|c|}{ 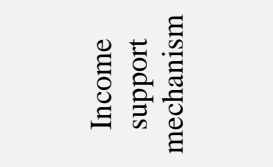 } & 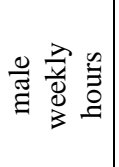 & 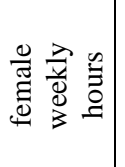 & 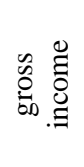 & 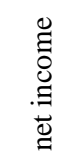 & 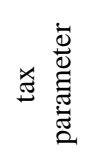 & 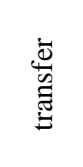 & $\stackrel{\Xi}{\stackrel{\Xi}{\Xi}}$ & 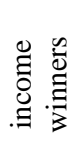 & 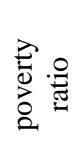 & 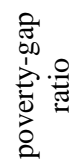 & 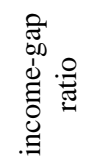 & 3 & $\begin{array}{l}3 \\
\vdots \\
\vdots \\
a\end{array}$ \\
\hline \multicolumn{3}{|c|}{ CURRENT } & 39.35 & 19.45 & 2930 & 2191 & -- & 101 & -- & -- & 4.23 & 0.58 & 13.71 & 94255 & 90047 \\
\hline GMI & $\mathrm{F}$ & 0.5 & 39.30 & 19.29 & 2925 & 2204 & 0.31 & 193 & 0.54 & 0.60 & 3.09 & 0.32 & 10.40 & 94254 & 91181 \\
\hline GMI & $\mathrm{F}$ & 0.75 & 39.25 & 19.13 & 2911 & 2197 & 0.37 & 269 & 0.53 & 0.60 & 0.93 & 0.06 & 6.82 & 94257 & 93332 \\
\hline GMI & $\mathrm{F}$ & 1 & 39.20 & 18.95 & 2892 & 2187 & 0.45 & 356 & 0.47 & 0.54 & 0.01 & 0.00 & 0.24 & 94255 & 94250 \\
\hline GMI & $\mathrm{P}$ & 0.5 & 39.29 & 19.31 & 2923 & 2200 & 0.01 & 193 & 0.64 & 0.66 & 2.50 & 0.26 & 10.49 & 94275 & 91790 \\
\hline GMI & $\mathrm{P}$ & 0.75 & 39.25 & 19.13 & 2906 & 2192 & 0.05 & 269 & 0.58 & 0.60 & 0.84 & 0.05 & 5.89 & 94268 & 93431 \\
\hline GMI & $\mathrm{P}$ & 1 & 39.18 & 18.94 & 2880 & 2173 & 0.14 & 356 & 0.48 & 0.52 & 0.01 & 0.00 & 0.38 & 94246 & 94241 \\
\hline GMI+WS & $\mathrm{F}$ & 0.5 & 39.32 & 19.44 & 2933 & 2213 & 0.36 & 406 & 0.58 & 0.62 & 3.90 & 0.51 & 13.18 & 94263 & 90383 \\
\hline GMI+WS & $\mathrm{F}$ & 0.75 & 39.30 & 19.37 & 2927 & 2210 & 0.40 & 435 & 0.62 & 0.65 & 2.79 & 0.29 & 10.23 & 94269 & 91495 \\
\hline GMI+WS & $\mathrm{F}$ & 1 & 39.28 & 19.29 & 2918 & 2204 & 0.45 & 467 & 0.61 & 0.64 & 1.55 & 0.13 & 8.17 & 94272 & 92735 \\
\hline GMI+WS & $\mathrm{P}$ & 0.5 & 39.32 & 19.45 & 2930 & 2208 & 0.06 & 405 & 0.68 & 0.70 & 3.44 & 0.42 & 12.33 & 94284 & 90865 \\
\hline GMI+WS & $\mathrm{P}$ & 0.75 & 39.29 & 19.38 & 2923 & 2206 & 0.07 & 435 & 0.67 & 0.69 & 2.31 & 0.24 & 10.29 & 94284 & 91987 \\
\hline GMI+WS & $\mathrm{P}$ & 1 & 39.25 & 19.29 & 2908 & 2192 & 0.12 & 466 & 0.60 & 0.58 & 0.81 & 0.09 & 11.24 & 94266 & 93460 \\
\hline UBI & $\mathrm{F}$ & 0.5 & 39.26 & 19.28 & 2913 & 2205 & 0.41 & 568 & 0.61 & 0.63 & 0.88 & 0.06 & 7.08 & 94276 & 93404 \\
\hline UBI & $\mathrm{F}$ & 0.75 & 39.21 & 19.12 & 2895 & 2196 & 0.50 & 814 & 0.58 & 0.57 & 0.06 & 0.00 & 3.37 & 94282 & 94225 \\
\hline UBI & $F$ & 1 & 39.15 & 18.95 & 2872 & 2184 & 0.60 & 1060 & 0.54 & 0.56 & 0.00 & 0.00 & 0.00 & 94281 & 94281 \\
\hline UBI & $\mathrm{P}$ & 0.5 & 39.25 & 19.29 & 2907 & 2199 & 0.13 & 568 & 0.66 & 0.62 & 0.52 & 0.04 & 7.10 & 94298 & 93779 \\
\hline UBI & $\mathrm{P}$ & 0.75 & 39.19 & 19.12 & 2883 & 2186 & 0.23 & 814 & 0.61 & 0.59 & 0.04 & 0.00 & 3.01 & 94297 & 94261 \\
\hline UBI & $\mathrm{P}$ & 1 & 39.12 & 18.94 & 2856 & 2170 & 0.33 & 1060 & 0.57 & 0.58 & 0.00 & 0.00 & 0.00 & 94290 & 94290 \\
\hline UBI+WS & $\mathrm{F}$ & 0.5 & 39.31 & 19.42 & 2929 & 2213 & 0.38 & 598 & 0.60 & 0.65 & 3.32 & 0.40 & 12.10 & 94267 & 90966 \\
\hline UBI+WS & $\mathrm{F}$ & 0.75 & 39.29 & 19.34 & 2922 & 2210 & 0.42 & 720 & 0.63 & 0.66 & 1.97 & 0.18 & 8.97 & 94274 & 92320 \\
\hline UBI+WS & $\mathrm{F}$ & 1 & 39.26 & 19.26 & 2912 & 2206 & 0.47 & 843 & 0.62 & 0.62 & 0.73 & 0.05 & 6.48 & 94277 & 93550 \\
\hline UBI+WS & $\mathrm{P}$ & 0.5 & 39.30 & 19.44 & 2925 & 2209 & 0.09 & 598 & 0.70 & 0.70 & 2.53 & 0.26 & 10.17 & 94295 & 91781 \\
\hline UBI+WS & $\mathrm{P}$ & 0.75 & 39.26 & 19.37 & 2914 & 2208 & 0.11 & 720 & 0.69 & 0.65 & 0.90 & 0.07 & 7.69 & 94299 & 93408 \\
\hline $\mathrm{UBI}+\mathrm{WS}$ & $\mathrm{P}$ & 1 & 39.20 & 19.24 & 2891 & 2185 & 0.18 & 841 & 0.57 & 0.54 & 0.23 & 0.01 & 2.81 & 94266 & 94039 \\
\hline WS & $\mathrm{F}$ & 0.5 & 39.35 & 19.57 & 2940 & 2223 & 0.34 & 353 & 0.62 & 0.62 & 4.64 & 0.76 & 16.41 & 94268 & 89651 \\
\hline WS & $\mathrm{F}$ & 0.75 & 39.34 & 19.57 & 2938 & 2223 & 0.36 & 352 & 0.63 & 0.66 & 4.16 & 0.62 & 14.96 & 94274 & 90141 \\
\hline WS & $\mathrm{F}$ & 1 & 39.33 & 19.58 & 2936 & 2224 & 0.39 & 352 & 0.66 & 0.69 & 3.65 & 0.50 & 13.78 & 94283 & 90652 \\
\hline WS & $\mathrm{P}$ & 0.5 & 39.34 & 19.59 & 2938 & 2218 & 0.03 & 352 & 0.70 & 0.71 & 4.19 & 0.65 & 15.51 & 94290 & 90125 \\
\hline WS & $\mathrm{P}$ & 0.75 & 39.34 & 19.59 & 2937 & 2223 & 0.03 & 352 & 0.72 & 0.73 & 3.63 & 0.54 & 14.87 & 94295 & 90683 \\
\hline WS & $\mathrm{P}$ & 1 & 39.31 & 19.58 & 2930 & 2215 & 0.06 & 352 & 0.68 & 0.67 & 3.02 & 0.38 & 12.56 & 94283 & 91283 \\
\hline
\end{tabular}




\begin{tabular}{|c|c|c|c|c|c|c|c|c|c|c|c|c|c|c|c|}
\hline \multicolumn{16}{|c|}{ Table 10. Equilibrium: $\eta=-\infty$} \\
\hline \multicolumn{3}{|c|}{ (a) } & (b) & (c) & (d) & (e) & (f) & $(\mathrm{g})$ & (h) & (i) & (1) & (m) & (n) & (o) & (p) \\
\hline \multicolumn{3}{|c|}{ 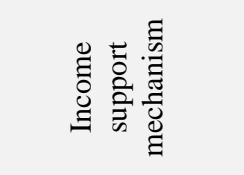 } & 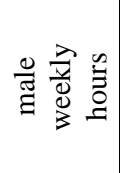 & 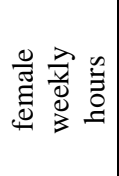 & 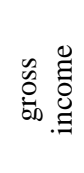 & 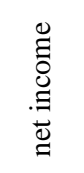 & 离 & 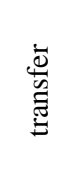 & 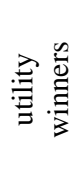 & 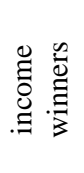 & 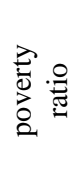 & 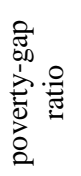 & 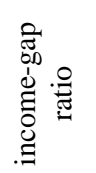 & 范 & 隹 \\
\hline \multicolumn{3}{|c|}{ Current } & 39.35 & 19.45 & 2930 & 2191 & -- & 101 & -- & -- & 4.23 & 0.58 & 13.71 & 94255 & 90047 \\
\hline GMI & $\mathrm{F}$ & 0.5 & 39.28 & 18.62 & 2899 & 2167 & 0.32 & 198 & 0.06 & 0.49 & 2.88 & 0.30 & 10.56 & 94031 & 91173 \\
\hline GMI & $\mathrm{F}$ & 0.75 & 39.19 & 17.83 & 2859 & 2135 & 0.39 & 281 & 0.04 & 0.42 & 0.76 & 0.05 & 6.03 & 93825 & 93077 \\
\hline GMI & $\mathrm{F}$ & 1 & 39.10 & 17.06 & 2816 & 2104 & 0.48 & 379 & 0.05 & 0.42 & 0.00 & 0.00 & 0.00 & 93628 & 93628 \\
\hline GMI & $\mathrm{P}$ & 0.5 & 39.27 & 18.65 & 2881 & 2163 & 0.02 & 197 & 0.07 & 0.54 & 2.39 & 0.25 & 10.34 & 94057 & 91683 \\
\hline GMI & $\mathrm{P}$ & 0.75 & 39.19 & 17.89 & 2841 & 2134 & 0.07 & 280 & 0.06 & 0.46 & 0.59 & 0.04 & 6.63 & 93852 & 93267 \\
\hline GMI & $\mathrm{P}$ & 1 & 39.08 & 17.08 & 2789 & 2091 & 0.17 & 378 & 0.06 & 0.42 & 0.00 & 0.00 & 0.00 & 93629 & 93629 \\
\hline GMI+WS & $\mathrm{F}$ & 0.5 & 39.31 & 18.82 & 2909 & 2178 & 0.37 & 405 & 0.07 & 0.53 & 3.88 & 0.51 & 13.03 & 94058 & 90213 \\
\hline GMI+WS & $\mathrm{F}$ & 0.75 & 39.25 & 18.20 & 2879 & 2153 & 0.41 & 436 & 0.05 & 0.47 & 2.55 & 0.26 & 10.35 & 93884 & 91358 \\
\hline GMI+WS & $\mathrm{F}$ & 1 & 39.19 & 17.49 & 2844 & 2125 & 0.46 & 471 & 0.04 & 0.41 & 1.25 & 0.11 & 8.57 & 93684 & 92449 \\
\hline GMI+WS & $\mathrm{P}$ & 0.5 & 39.30 & 18.84 & 2891 & 2173 & 0.06 & 405 & 0.08 & 0.61 & 3.35 & 0.42 & 12.44 & 94080 & 90752 \\
\hline GMI+WS & $\mathrm{P}$ & 0.75 & 39.25 & 18.19 & 2859 & 2150 & 0.08 & 435 & 0.07 & 0.52 & 2.13 & 0.22 & 10.45 & 93890 & 91785 \\
\hline GMI+WS & $\mathrm{P}$ & 1 & 39.16 & 17.47 & 2817 & 2111 & 0.14 & 470 & 0.05 & 0.42 & 0.76 & 0.08 & 10.33 & 93671 & 92918 \\
\hline UBI & $\mathrm{F}$ & 0.5 & 39.25 & 18.67 & 2891 & 2171 & 0.41 & 568 & 0.09 & 0.54 & 0.86 & 0.06 & 6.83 & 94071 & 93220 \\
\hline UBI & $\mathrm{F}$ & 0.75 & 39.16 & 17.92 & 2847 & 2139 & 0.52 & 814 & 0.08 & 0.49 & 0.04 & 0.00 & 3.59 & 93875 & 93840 \\
\hline UBI & $\mathrm{F}$ & 1 & 39.06 & 17.14 & 2800 & 2103 & 0.62 & 1060 & 0.08 & 0.49 & 0.00 & 0.00 & 0.00 & 93669 & 93669 \\
\hline UBI & $\mathrm{P}$ & 0.5 & 39.23 & 18.61 & 2865 & 2162 & 0.14 & 568 & 0.11 & 0.56 & 0.46 & 0.03 & 7.25 & 94070 & 93615 \\
\hline UBI & $\mathrm{P}$ & 0.75 & 39.13 & 17.86 & 2818 & 2127 & 0.24 & 814 & 0.10 & 0.53 & 0.02 & 0.00 & 2.35 & 93870 & 93850 \\
\hline UBI & $\mathrm{P}$ & 1 & 39.02 & 17.07 & 2765 & 2087 & 0.35 & 1060 & 0.09 & 0.51 & 0.00 & 0.00 & 0.00 & 93659 & 93659 \\
\hline UBI+WS & $\mathrm{F}$ & 0.5 & 39.30 & 18.78 & 2905 & 2178 & 0.38 & 595 & 0.08 & 0.55 & 3.29 & 0.39 & 11.76 & 94057 & 90789 \\
\hline UBI+WS & $\mathrm{F}$ & 0.75 & 39.24 & 18.10 & 2872 & 2152 & 0.43 & 715 & 0.07 & 0.47 & 1.95 & 0.17 & 8.48 & 93866 & 91932 \\
\hline UBI+WS & $\mathrm{F}$ & 1 & 39.17 & 17.40 & 2836 & 2125 & 0.48 & 835 & 0.06 & 0.42 & 0.70 & 0.04 & 5.76 & 93669 & 92982 \\
\hline UBI+WS & $\mathrm{P}$ & 0.5 & 39.29 & 18.85 & 2886 & 2175 & 0.10 & 595 & 0.10 & 0.61 & 2.58 & 0.26 & 10.01 & 94096 & 91537 \\
\hline UBI+WS & $\mathrm{P}$ & 0.75 & 39.22 & 18.19 & 2850 & 2151 & 0.12 & 715 & 0.10 & 0.54 & 0.87 & 0.07 & 7.54 & 93904 & 93046 \\
\hline UBI+WS & $\mathrm{P}$ & 1 & 39.12 & 17.46 & 2803 & 2107 & 0.19 & 833 & 0.07 & 0.44 & 0.09 & 0.00 & 4.17 & 93676 & 93589 \\
\hline WS & $\mathrm{F}$ & 0.5 & 39.33 & 18.96 & 2900 & 2187 & 0.34 & 350 & 0.06 & 0.54 & 4.74 & 0.77 & 16.28 & 94066 & 89366 \\
\hline WS & $\mathrm{F}$ & 0.75 & 39.29 & 18.40 & 2874 & 2167 & 0.37 & 348 & 0.06 & 0.51 & 4.08 & 0.62 & 15.15 & 93889 & 89854 \\
\hline WS & $\mathrm{F}$ & 1 & 39.26 & 17.84 & 2848 & 2147 & 0.40 & 345 & 0.05 & 0.46 & 3.71 & 0.50 & 13.57 & 93720 & 90052 \\
\hline WS & $\mathrm{P}$ & 0.5 & 39.33 & 18.91 & 2896 & 2181 & 0.04 & 350 & 0.07 & 0.62 & 4.21 & 0.64 & 15.23 & 94068 & 89888 \\
\hline WS & $\mathrm{P}$ & 0.75 & 39.29 & 18.31 & 2868 & 2162 & 0.04 & 347 & 0.06 & 0.56 & 3.76 & 0.54 & 14.37 & 93875 & 90148 \\
\hline WS & $\mathrm{P}$ & 1 & 39.23 & 17.73 & 2836 & 2133 & 0.07 & 344 & 0.05 & 0.43 & 2.96 & 0.38 & 12.83 & 93684 & 90755 \\
\hline
\end{tabular}




\section{Appendix A. The microecometric model}

\section{A.1. Household behaviour}

The basic modelling framework belongs to the family of the Random Utility models and is similar to the one adopted in a series of papers by Aaberge et al. $(1995,1999,2000 \mathrm{a}, 2000 \mathrm{~b}, 2004,2011)$ and Colombino et al. (2010). ${ }^{11}$ We will consider households with two decision-makers (couples) or one decision-maker (singles). In both cases the decisionmakers are aged $20-55$ and are not retired or students. Of course there might be other people in the household, but their behaviour is taken as exogenous.

A couple $n$ is assumed to solve the following problem

$$
\text { (A.1) } \begin{array}{ll} 
& h_{F} \in A \\
& h_{M} \in A \\
& C=R\left(w_{F}^{n} h_{F}, w_{M}^{n} h_{M}, y^{n}\right)
\end{array}
$$

where

$U^{n}\left(C, h_{F}, h_{M}, \varepsilon\right)=$ utility function $h_{g}=$ average weekly hours of work required by the chosen job in the choice set for partner of gender $g=\mathrm{F}$ (female) or M (male);

$A=$ set of 12 discrete values (see section A.3);

$w_{g}^{n}=$ hourly wage rate of partner $g$;

$y^{n}=$ vector of exogenous household gross incomes;

$C=$ net disposable household income;

$\varepsilon=$ random variable that captures the effect of unobserved characteristics of the household-job match;

$R=$ tax-transfer rule that transforms gross incomes into net available household income.

The first two constraints of problem (A.1) say that the hours of work $h_{g}$ are chosen within a discrete set of values $A$ including also 0 hours. This discrete set of values can be interpreted as the actual choice set (maybe determined by institutional constraints) or as approximations to the true (possibly continuous) choice set.

The third constraint says that net income $C$ is the result of a tax-transfer rule $R$ applied to gross incomes.

\footnotetext{
${ }^{11}$ Surveys of various approaches to modelling labour supply for tax reform simulation are provided by Creedy et al. (2005), Bourguignon et al. (2006) and Meghir et al. (2008).
} 
We write the utility function $U^{n}\left(C, h_{F}, h_{M}, \mathcal{E}\right)$ as the sum of a systematic part and a random component:

$$
\mathrm{U}^{n}\left(C, h_{F}, h_{M}, \mathcal{\varepsilon}\right)=V\left(R\left(w_{F}^{n} h_{F}, w_{M}^{n} h_{M}, y^{n}\right), h_{F}, h_{M}, Z^{n} ; \theta\right)+\varepsilon
$$

where $Z^{n}$ is a vector of household characteristics and $\theta$ is a vector of parameters to be estimated. The interpretation of the random variable $\varepsilon$ is analogous to the one given by McFadden in his presentations of the Conditional Logit model (McFadden, 1974): besides the observed variables, there are characteristics of the household-job match that are observed by the household but not by the econometrician; the random variable $\varepsilon$ is meant to account for the contribution to utility by those characteristics. ${ }^{12}$ Under the assumption that $\varepsilon$ is i.i.d. extreme value, it is well known that the probability that household $n$ subject to tax-transfer regime $R$ chooses $h_{F}=f, h_{M}=m$ is given by

$$
P^{n}(f, m ; \theta, R)=\frac{\exp \left\{V\left(R\left(w_{F}^{n} f, w_{M}^{n} m, y^{n}\right), f, m, Z^{n} ; \theta\right)\right\}}{\sum_{h_{F} \in \Omega} \sum_{h_{M} \in \Omega} \exp \left\{V\left(R\left(w_{F}^{n} h_{F}, w_{M}^{n} h_{M}, y^{n}\right), h_{F}, h_{M}, Z^{n} ; \theta\right)\right\}}
$$

In a similar way, a single $s$ of gender $g$ is assumed to solve a constrained utility maximization problem as follows:

$$
\max _{C, h, \varepsilon} U_{g}^{s}(C, h, \varepsilon)
$$

(A.4) $h \in A$

$$
C=R\left(w^{s} h, y^{s}\right)
$$

where

$h=$ average weekly hours of work required by the chosen job.

In this case, the utility function $U_{g}^{s}(C, h, \varepsilon)$ can be written as the sum of a systematic part and a random component:

$$
U_{g}^{s}(C, h ; \varepsilon)=V\left(R\left(w^{s} h, y^{s}\right), h, Z^{s} ; \theta_{g}\right)+\varepsilon
$$

\footnotetext{
${ }^{12}$ Most of the labour supply literature adopting the Conditional Logit framework tends to privilege a different interpretation of the random component, where the true utility function is just the systematic component $V$ of expression (A.2) and the random variable $\mathcal{E}$ is an optimization error (e.g. Van Soest 1995 and Duncan and Giles 1996). An implication of this interpretation is that the econometrician is assumed to know more than the household itself: the econometrician knows that the true utility is $V$, while the households base their choices on a wrong utility level $U$. We find this interpretation less acceptable than the one originally proposed by McFadden, so here we follow the latter. The interpretation we adopt, however, implies that we cannot test for the (local) quasi-concavity of the utility function: we estimate $V$, and we could make a test on $V$, but the true utility function is not $V$ but $U$, and $U$ is a function of an unknown random variable $\mathcal{E}$.
} 


\section{A.2. Empirical specification of preferences}

We choose a quadratic specification since it is linear-in-parameters and it represents a good compromise between

flexibility and ease of estimation:

$$
\begin{aligned}
& V^{n}=\theta_{C} C+\theta_{F}\left(T-h_{F}\right)+\theta_{M}\left(T-h_{M}\right)+ \\
& \quad+\theta_{C C} C^{2}+\theta_{F F}\left(T-h_{F}\right)^{2}+\theta_{M M}\left(T-h_{M}\right)^{2}+ \\
& \quad+\theta_{C F} C\left(T-h_{M}\right)+\theta_{C M} C\left(T-h_{M}\right)+\theta_{F M}\left(T-h_{F}\right)\left(T-h_{M}\right) \\
& V^{s}=\theta_{C} C+\theta_{g}\left(T-h_{g}\right)+\theta_{C C} C^{2}+\theta_{g g}\left(T-h_{g}\right)^{2}+\theta_{C g} C\left(T-h_{g}\right)
\end{aligned}
$$

where $V^{n}$ and $V^{s}$ denote the systematic part of the utility function respectively for couples and singles and $T$ denotes total available time.

Some of the above parameters $\theta s$ are made dependent on characteristics:

$$
\begin{aligned}
\theta_{F}= & \beta_{F 0}+\beta_{F 1}(\text { Age of the wife })+\beta_{F 2}(\text { Age of the wife })^{2}+ \\
& +\beta_{F 3}(\# \text { Children })+\beta_{F 4}(\# \text { Children under } 6)+\beta_{F 5}(\# \text { Children } 6-10) \\
\theta_{M}= & \beta_{M 0}+\beta_{M 1}(\text { Age of the husband })+\beta_{M 2}(\text { Age of the husband })^{2}+ \\
& +\beta_{M 3}(\# \text { Children })+\beta_{M 4}(\# \text { Children under } 6)+\beta_{M 5}(\# \text { Children 6-10) } \\
\theta_{g}= & \beta_{g 0}+\beta_{g 1}(\text { Age })+\beta_{g 2}(\text { Age })^{2}+\beta_{g 3}(\# \text { Children })+ \\
& +\beta_{g 4}(\# \text { Children under } 6)+\beta_{g 5}(\# \text { Children 6-10) } \\
\theta_{C}= & \beta_{C 0}+\beta_{C 1}(\text { Household's size }) .
\end{aligned}
$$

Notice that the parameters are separately estimated for couples, single females and single males.

\section{A.3. Empirical specification of the opportunity sets}

We assume that each partner in a couple household can choose between 10 values (from 1 to 80) of weekly hours of work. Each value is randomly drawn from one of the following ten intervals: 1-8, 9-16, 17-24, 25-32, 33-40, 41-48, 49-56, 5764, 65-72, 73-80. Moreover they can also choose to be out-of-work but we don't distinguish between activity and unemployment status (therefore there is one alternative with zero hours of work). Thus each couple household chooses among 121 alternatives. In order to compute net household income $C$ for each one of the household jobs contained in $A \times A$, we use the EUROMOD Microsimulation model. ${ }^{13}$ In other words EUROMOD mimics the tax-transfer rule R. Wage rates for those who are observed as not employed are imputed on the basis of a wage equation estimated on the

\footnotetext{
${ }^{13}$ EUROMOD is a tax-benefit microsimulation model for the European Union that enables researchers and policy analysts to calculate, in a comparable manner, the effects of taxes and benefits on household incomes and work incentives for the population of each country and for the EU as a whole. EUROMOD was originally designed by a research team under the direction of Holly Sutherland at the Department of Economics in Cambridge, UK. It is now developed and updated at the Microsimulation Unit at ISER (University of Essex, UK).
} 
employed subsample and corrected for sample selection. For the single households, we assume that the single household head can choose among the same set of 11 alternatives as where each partner of a couple household chooses. ${ }^{14}$

The data typically show a more or less pronounced concentration of people around hours corresponding to full-time, parttime and non-working. The models of the type outlined above are typically unable to reproduce these peaks. A useful procedure consists of adding alternative-specific dummies. We define the following dummies for part-time, full-time, overtime, working and not working (the excluded condition being "working less than 17 weekly hours"):

(A.9) $D_{g 3}\left(h_{g}\right)=\left\{\begin{array}{l}1 \text { if } 49 \leq h_{g} \\ 0 \text { otherwise }\end{array}\right.$

$$
\begin{aligned}
D_{g 1}\left(h_{g}\right) & =\left\{\begin{array}{l}
1 \text { if } 17 \leq h_{g} \leq 32 \\
0 \text { otherwise }
\end{array}\right. \\
D_{g 2}\left(h_{g}\right) & =\left\{\begin{array}{l}
1 \text { if } 33 \leq h_{g} \leq 48 \\
0 \text { otherwise }
\end{array}\right.
\end{aligned}
$$

$$
D_{g 4}\left(h_{g}\right)=\left\{\begin{array}{l}
1 \text { if } 0<h_{g} \\
0 \text { otherwise }
\end{array}\right.
$$

with $\mathrm{g}=\mathrm{F}$ (female) or M (male).

Aaberge et al. $(1995,1999)$ and Dagsvik (2000) provide a formal justification of this procedure: assuming a non-uniform probability density function of the alternatives in the opportunity set and adopting an appropriate empirical specification allows rewriting the choice probabilities respectively for couple and single households as follows:

$$
P^{n}(f, m ; \theta, \gamma, R)=\frac{\exp \left\{V\left(R\left(w_{F}^{n} f, w_{M}^{n} m, y^{n}\right), f, m, Z^{n} ; \theta\right)+\sum_{k=1}^{4} \gamma_{F k} D_{F k}(f)+\sum_{k=1}^{4} \gamma_{M k} D_{M k}(m)\right\}}{\sum_{h_{k} \in \Omega} \sum_{h_{u} \in \Omega} \exp \left\{V\left(R\left(w_{F}^{n} h_{F}, w_{M}^{n} h_{M}, y^{n}\right), h_{F}, h_{M}, Z^{n} ; \theta\right)+\sum_{k=1}^{4} \gamma_{F k} D_{F k}\left(h_{F}\right)+\sum_{k=1}^{4} \gamma_{M k} D_{M k}\left(h_{M}\right)\right\}}
$$

$$
P^{s}\left(j ; \theta_{g}, \gamma_{g}, R\right)=\frac{\exp \left\{V\left(R\left(w_{g}^{s} j, y^{s}\right), g, Z^{s} ; \theta\right)+\sum_{k=1}^{4} \gamma_{g k} D_{k}(j)\right\}}{\sum_{h \in \Omega} \exp \left\{V\left(R\left(w_{s}^{s} h, y^{s}\right), h, Z^{s} ; \theta\right)+\sum_{k=1}^{4} \gamma_{g k} D_{k}(h)\right\}}
$$

where the $\gamma^{\prime}$ s and the $\theta$ 's are parameters to be estimated. The coefficients $\gamma^{\prime}$ s will reflect, possibly besides other costs or utility components, the different availability or density of different types of jobs. This interpretation of the dummies entails an interpretation of the model as representing a matching process (between types of household and types of jobs) rather than simply a labour supply decision and forms the basis for a simulation procedure that accounts for market equilibrium (see section A.7).

\footnotetext{
${ }^{14}$ A comparison and evaluation of different procedures to specify the choice set is provided by Aaberge et al. (2009).
} 


\section{A.4. Estimates}

The parameters are estimated by Maximum Likelihood. For the estimation and simulation exercise presented in this paper we use the Italian dataset generated by EUROMOD team from the Survey of Household Income and Wealth (SHIW) 1998.

The inclusion criteria (rather common in the literature on behavioural evaluation of tax reforms) are as follows:

- Couple and single households;

- Employed (self-employed included), unemployed or inactive (students and disabled are excluded);

- Both partners of couple households and heads of single households aged 20 - 55.

The estimates based on the sample of couples, single men and single women (respectively 2955, 291 and 366 observations) are reported in Table A.1.

The crucial preference parameters are:

$\beta_{C 0}$ and $\theta_{C C}$ (related to the marginal utility of income);

$\beta_{F 0}$ and $\theta_{F F}$ (related to the marginal utility of wife's leisure);

$\beta_{M 0}$ and $\theta_{M M}$ (related to the marginal utility of husband's leisure).

$\beta_{g 0}$ and $\theta_{g g}$ (related to the marginal utility of single household head leisure).

The other parameters $\beta^{\prime} s$ and $\theta^{\prime} s$ measure the effects of various interactions of leisure times and income among themselves and with household characteristics.

The marginal utility of income and the marginal utility of wife's and husband's leisure appear to be positive and decreasing (at least at the observed choices).

The wife's and the husband's leisure appear to be complements, in the sense that more leisure of one of them has a positive effect on the marginal utility of leisure of the other one. 
Table A.1. Parameter estimates

\begin{tabular}{|c|c|c|c|}
\hline & Couple & Single female & Single Male \\
\hline$\beta_{F 0}$ & $.3301752 * * *$ & .1562657 & \\
\hline$\beta_{F 1}$ & $-.0077954 * * *$ & $-.0085422^{*}$ & \\
\hline$\beta_{F 2}$ & $.0001051^{* * *}$ & $.0001062 *$ & \\
\hline$\beta_{F 3}$ & $.0086118^{* * *}$ & .0097963 & \\
\hline$\beta_{F 4}$ & -.0018444 & -.0025955 & \\
\hline$\beta_{F 5}$ & .0030899 & .0130587 & \\
\hline$\beta_{M 0}$ & .0338491 & & $.2237299 *$ \\
\hline$\beta_{M 1}$ & .001687 & & -.0053004 \\
\hline$\beta_{M 2}$ & -.0000218 & & .0000694 \\
\hline$\beta_{M 3}$ & .0035718 & & -.0685087 \\
\hline$\beta_{M 4}$ & $-.0105606^{* * * *}$ & & .0614548 \\
\hline$\beta_{M 5}$ & $-.0077151 * *$ & & .0634671 \\
\hline$\beta_{C 0}$ & $.0004311^{* * *}$ & -.0001394 & .0002968 \\
\hline$\beta_{C 1}$ & -.0000251 & .0000433 & -.0000642 \\
\hline$\theta_{C C}$ & $-9.12 \mathrm{e}-09^{*}$ & $-1.42 e-08$ & $-8.87 e-09$ \\
\hline$\theta_{F F}$ & $-.0008251 * * *$ & $.0008978^{*}$ & \\
\hline$\theta_{M M}$ & $.0003973 * *$ & & -.0000417 \\
\hline$\theta_{C F}$ & $-1.92 \mathrm{e}-06^{*}$ & $5.70 \mathrm{e}-06$ & \\
\hline
\end{tabular}


Table A.1. Parameter estimates (cont'd)

\begin{tabular}{|l|l|l|l|}
\hline & Couple & Single female & Single Male \\
\hline$\theta_{C M}$ & $-1.01 \mathrm{e}-06$ & & $-1.23 \mathrm{e}-06$ \\
\hline$\theta_{F M}$ & $.0001992^{*}$ & & \\
\hline$\gamma_{F 1}$ & $3.07818^{* * *}$ & $4.069606 * * *$ & \\
\hline$\gamma_{F 2}$ & $5.223014 * * *$ & $7.077753^{* * *}$ & \\
\hline$\gamma_{F 3}$ & $5.260581^{* * *}$ & $6.363261 * * *$ & \\
\hline$\gamma_{F 4}$ & $-3.356024 * * *$ & $-1.131054 * *$ & $2.997396 * * *$ \\
\hline$\gamma_{M 1}$ & $3.673685^{* * *}$ & & $6.786832 * * *$ \\
\hline$\gamma_{M 2}$ & $8.314315^{* * *}$ & & $7.232927 * * *$ \\
\hline$\gamma_{M 3}$ & $8.917805^{* * *}$ & & -.7926529 \\
\hline$\gamma_{M 4}$ & $-.8084671^{* * *}$ & & \\
\hline $\begin{array}{l}\text { For the meaning of the coefficient symbols see expressions A.6, A.7 and A.8. } \\
* * * \\
* * \\
*\end{array}=$ significance $<1 \%$ & & \\
$* *$ significance $<5 \%$ & & \\
\hline
\end{tabular}




\section{A.5. Behavioural simulation method}

The estimated model is used to simulate the effects of alternative hypothetical tax-transfer reforms. Suppose we are interested in some tax-transfer rule $\mathrm{R}$. We explain the procedure with reference to the case of couples. Let $P^{n}(f, m ; \theta, \gamma, R)$ be the probability that couple household $n$ chooses $(f, m)$ under the $R$ tax-transfer regime, computed on the basis of the estimated parameters. Suppose we are interested in simulating the expected value of some function $\psi^{n}(f, m)$ : it might be the net available income under the new rule, hours worked, taxes paid etc. Then we compute the expected value of that variable after the policy is implemented as follows:

$$
E\left(\psi^{n}(f, m)\right)=\sum_{f \in \Omega} \sum_{m \in \Omega} \psi^{n}(f, m) P^{n}\left(f, m, Z^{n} ; \theta, \gamma, R\right) .
$$

A similar procedure is used to simulate the effects of alternative tax-transfer reforms for singles.

\section{A.6. Social evaluation}

In section 3 of the main text we define two Social Welfare functions. Their computation requires the following steps.

1) Expected maximum utility attained by household $n$ under tax-transfer regime $(R)::^{15}$

(A.12)

$$
v^{n}(R)=\left\{\begin{array}{l}
\ln \left(\sum_{h_{F} \in \Omega} \sum_{h_{M} \in \Omega} \exp \left\{V\left(R\left(w_{F}^{n} h_{F}, w_{M}^{n} h_{M}, y^{n}\right), h_{F}, h_{M}, Z^{n} ; \theta, \gamma\right)+\sum_{k=1}^{5} \gamma_{F k} D_{F k}\left(h_{F}\right)+\sum_{k=1}^{5} \gamma_{F k} D_{M k}\left(h_{M}\right)\right\}\right) \\
\text { if couple } \\
\ln \left(\sum_{h \in \Omega} \exp \left\{V\left(R\left(w^{n} h, y^{n}\right), h, Z^{n} ; \theta, \gamma\right)+\sum_{k=1}^{5} \gamma_{k} D_{k}(h)+\sum_{k=1}^{5} \gamma_{k} D_{k}(h)\right\}\right) \\
\text { if single }
\end{array}\right.
$$

2) Interpersonally-comparable-metric utility ${ }^{16}$ of household $i$ under tax regime $R, \mu_{i}(R)$.

Let $v^{0}\left(R_{0}\right)$ be the expected maximum utility attained by a reference household under a reference tax-transfer regime. In this paper we choose as reference household the poorest single in 1998 and as reference tax-transfer system the 1998 system:

$$
v^{0}\left(R_{0}\right)=\ln \left(\sum_{h \in \Omega} \exp \left\{V\left(R_{0}\left(w^{0} h, y^{0}\right), h, Z^{0} ; \theta, \gamma\right)+\sum_{k=1}^{5} \gamma_{k} D_{k}(h)+\sum_{k=1}^{5} \gamma_{k} D_{k}(h)\right\}\right)
$$

The interpersonally-comparable money-metric utility of household $n$ under tax regime $R, \mu^{n}(R)$, is then defined by:

$$
\ln \left(\sum_{h \in \Omega} \exp \left\{V\left(\mu^{n}(R), h, Z^{0} ; \theta, \gamma\right)+\sum_{k=1}^{5} \gamma_{k} D_{k}(h)+\sum_{k=1}^{5} \gamma_{k} D_{k}(h)\right\}\right)=v^{n}(R) .
$$

\footnotetext{
${ }^{15}$ For the derivation of this expression, see McFadden (1978) and Ben-Akiva et al. (1985). The same methodology for empirical welfare evaluation is used by Colombino (1998).

${ }^{16}$ A comprehensive explanation of the procedure adopted for developing interpersonally comparable measures of utility is provided by King (1983).
} 
In other words, $\mu^{n}(R)$ is the net available income needed by the reference household under the reference tax-transfer regime in order to attain the same expected maximum utility level of household $n$ under tax-transfer regime $R$.

3) Expressions (A.12) - (A.14) assume that the household is able to choose the constrained utility-maximizing "job". In the Non-behavioural simulation this assumption is not appropriate anymore. The procedure we adopt is explained hereafter; it is referred to single households, the extension to couples is immediate.

Let

$\bar{h}^{n}=$ hours of work of household $n$ under the 1998 regime,

$\bar{h}^{0}=$ hours of work of the reference household under the 1998 regime.

Then $\mu^{n}(R)$ is defined by:

$$
V\left(\mu^{n}(R), \bar{h}^{0}, Z^{0} ; \theta, \gamma\right)=V\left(R\left(w^{n} \bar{h}^{n}, y^{n}\right), h^{n}, Z^{n} ; \theta, \gamma\right) .
$$

4) The Gini Social Welfare (GSW) function and the Poverty-adjusted Social Welfare (PAGSW) function are computed as follows:

$$
G S W(R)=\mu(R)(1-I(R))
$$

(A.16)

$$
\operatorname{PAGSW}(R)=\mu(R)(1-I(R)-p(R))
$$

where

$\mu(R)=\frac{1}{N} \sum_{n} \mu^{n}(R)$

$I(R)=$ Gini coefficient of the sample distribution of $\mu_{n}(R)$

$p(R)=$ head-count poverty ratio. 


\section{Appendix B. Simulation under equilibrium}

The microeconometric model illustrated in Appendix A adopts the widely used refinement consisting of introducing alternative-specific constants, which should account for a number of factors such as the different density or accessibility of different types of jobs, search or fixed costs and systematic utility components otherwise not accounted for (expression A.10). Many papers have adopted a similar procedure, e.g.: Van Soest (1995), Aaberge et al. (1995, 1999, 2011a), Kalb (2000), Dagsvik and Strøm (2006), Kornstad et al. (2007) and Colombino et al. (2010). All the authors adopting the "dummies refinement" so far have performed the simulations while leaving the dummies' coefficients $\gamma$ 's unchanged. The policy simulation is most commonly interpreted as a comparative statics exercise, where different equilibriainduced by different tax-transfer regimes - are compared. We claim that the standard procedure in general is not consistent with the comparative statics interpretation. According to a basic notion of equilibrium, the number of people willing to work must equal to the number of available jobs. Since the $\gamma$ 's reflect - at least in part - the number and the composition of available jobs, and since the number of people willing to work and their distribution across different job types in general change as a consequence of the reforms, it follows that in general the $\gamma$ 's must also change. A series of papers by Aaberge et al. (1995, 1999, 2011a), building on a matching model developed by Dagsvik (1994, 2000), extend the basic random utility approach to include a random choice set and provide a structural interpretation of the "dummies refinement" that leads very naturally to a simulation procedure consistent with comparative statics. ${ }^{17}$

For simplicity of exposition we consider here a single individual. The probability that a job of type $j$ is chosen is

$$
P(j ; \theta, \gamma, R)=\frac{\exp \left\{V(R(w j, y), j ; Z, \theta)+\sum_{k=1}^{4} \gamma_{k} D_{k}(j)\right\}}{\sum_{h \in A} \exp \left\{V(R(w h, y), h ; Z, \theta)+\sum_{k=1}^{4} \gamma_{k} D_{k}(h)\right\}}
$$

It can then be shown (Aaberge and Colombino 2011a) that - under certain assumptions - the coefficients of the dummy variables have the following interpretation:

$$
\gamma_{4}=\ln \left(\frac{J}{H}\right)
$$

and

$$
\gamma_{k}=\ln \left(\frac{J_{k} / J}{A_{k}}\right), k=1,2,3
$$

\footnotetext{
${ }^{17}$ A different procedure for equilibrium simulation - which however would not be appropriate with our microeconometric model - has been proposed by Creedy and Duncan (2001). The possible inconsistency of the Creedy-Duncan procedure when using a model of the type we adopt was suggested to me by Lennart Flood.
} 
where $J=$ number of market jobs (i.e. opportunities with $h>0$ ), $J_{1}$ is the number of jobs with $17 \leq h \leq 32, J_{2}$ is the number of jobs with $33 \leq h \leq 48$ and $J_{3}$ is the number of jobs with $49 \leq h$. $H$ and $A_{k}$ are normalizing constants that account for the presence of factors other than jobs density (such as search or fixed costs, number of non-market opportunities etc.).

To further simplify the exposition we assume now that only the dummy $D_{4}$ is introduced, i.e. we rewrite the choice probability as follows: ${ }^{18}$

$$
P(j ; \theta, \gamma, R)=\frac{\exp \left\{V(R(w j, y), j ; Z, \theta)+\gamma_{4} D_{4}(j)\right\}}{\sum_{h \in A} \exp \left\{V(R(w h, y), h ; Z, \theta)+\gamma_{4} D_{4}(h)\right\}}
$$

Let us assume a constant-elasticity labour demand function

$$
J=K \bar{w}^{\eta}
$$

where $\bar{w}$ is the average wage rate, $K$ is a constant and $\eta$ is the wage elasticity of the demand for labour. In this paper we present simulation results based on alternative values of $\eta$. Notice that given $\eta$ and the observed pre-reform number of employed (and - in equilibrium - jobs) $J$ we can retrieve $K$. Moreover, given the estimate of $\gamma_{4}$, we can retrieve $H$ from (B.2). Let us write $w=\bar{w}+u$.

Using B.2 and B.5 we write:

$$
\gamma_{4}=\ln \left(\frac{K \bar{w}^{\eta}}{H}\right) \equiv \gamma(\bar{w})
$$

We then define $\left.\pi_{i}(R, \bar{w})\right)$ as the probability that individual $i$ is working given tax-transfer regime $\mathrm{R}$ and average wage rate $\bar{w}_{T}:$

$$
\pi_{i}(R, \bar{w}, \gamma(\bar{w})) \equiv \sum_{g>0} \frac{\exp \left\{V\left(R\left(\bar{w}+u_{i}, g, y\right), g ; Z, \theta\right)+\gamma(\bar{w}) D_{4}(g)\right\}}{\sum_{h \in A} \exp \left\{V\left(R\left(\bar{w}+u_{i}, h, y\right), h ; Z, \theta\right)+\gamma(\bar{w}) D_{4}(h)\right\}}
$$

where $\bar{w}+u_{i}=w_{i T}$. Assuming that the choices under the tax-transfer regime $R$ corresponds are in equilibrium, we must have:

\footnotetext{
${ }^{18}$ The extension to couples and to the multi-dummy case is explained in Colombino (2010).
} 


$$
\left.\sum_{i} \pi_{i}\left(R, \bar{w}_{R}, \gamma\left(\bar{w}_{R}\right)\right)\right)=K \bar{w}_{R}{ }^{\eta} .
$$

where $\bar{w}_{R}$ denotes the mean of the equilibrium wage distribution.

\section{Perfectly rigid demand}

In the special case of a perfectly rigid demand (zero elasticity), the number of jobs remains fixed but the wage rate must be adjusted so that the number of people willing to work under the new regime is equal to the pre-reform number of jobs:

$$
\sum_{i} \pi_{i}\left(R, \bar{w}_{R}, \gamma\left(\bar{w}_{R}\right)\right)=J .
$$

\section{Perfectly elastic demand}

When the demand for labour is perfectly elastic, the market is always in equilibrium at the initial wage rate. However, since the number of working people in general will change under a new tax-transfer rule and since the number of jobs in equilibrium must be equal to the number of people willing to work, it follows that the parameter $\gamma_{4}=\ln \left(\frac{J}{H}\right)$ must change. Then the equilibrium condition is

$$
J=H e^{\gamma_{4}} .
$$

where $J$ is the current (observed or simulated) number of employed. In this case $\bar{w}$ remains unchanged, while instead $\gamma_{4 R}$ must be directly adjusted so as to fulfil condition (B.11). The case with fixed wage rate and the demand absorbing any change in supply at that wage, actually corresponds to the scenario implicitly assumed in most tax-transfer simulations. However those simulations are not consistent since they do not take condition (B.11) into account.

\section{Equiproportional changes in $\mathrm{J}$ and $\mathrm{H}$.}

One might consider a particular scenario in which $J$ and $H$ change in the same proportion as a consequence of a reform. For example one might argue that the economy is organized so that there is a fixed proportion between the dimension of the labour market and the dimension of the "leisure" economy. ${ }^{19}$ Clearly under this assumption the coefficient $\gamma_{4}$ should be left unchanged. The simulation that in the main text we label as No Equilibrium could also be interpreted as corresponding to this scenario.

\footnotetext{
${ }^{19}$ This possibility was suggested by Rolf Aaberge in a comment to a previous version of this paper.
} 


\section{Appendix C. The reforms}

Definitions:

$x_{F}=w_{F} h_{F}=$ female gross earnings

$x_{M}=w_{M} h_{M}=$ male gross earnings

$x=x_{F}+x_{M}$

$y_{F}=$ female unearned gross income

$y_{M}=$ male unearned gross income

$m=$ other household net income

$S_{F}=$ social security contributions (female)

$S_{M}=$ social security contributions (male)

$S=S_{F}+S_{M}$

$I_{F}=g_{F}+y_{F}-S_{F}=$ taxable income (female)

$I_{M}=g_{M}+y_{M}-S_{M}=$ taxable income (male)

$I=I_{F}+I_{M}$

$P=$ poverty line

$N=$ number of people in the household

$G=\alpha P \sqrt{N}$ with $\alpha=1,0.75,0.50$

$C_{F}=$ net disposable income (female)

$C_{M}=$ net disposable income (male)

$C=m+C_{F}+C_{M}$

$T=$ taxes paid by the household

$B=$ benefits or transfers received by household

$\mathrm{q}=$ average propensity to consumption

$r=$ average VAT rate

$\omega=$ proportional subsidy on the gross wage rate $=0.1$

$\varphi()=$. progressive tax function (from gross income to net income). The current (1998) marginal tax rates are as follows: 
Income Brackets Marginal Tax Rates

$\begin{array}{cc}0-15 & 18 \\ 15-30 & 26 \\ 30-60 & 33 \\ 60-135 & 39 \\ >135 & 45\end{array}$

Income brackets are in Millions of Lire (10 Millions of Lire $=5165$ Euros).

Under the 1998 system the above rates are applied to personal earnings, together with deductions, allowances and benefits. Under the reforms all deductions, tax credits and benefits are cancelled, the income brackets are kept unchanged and the marginal tax rates (either the flat or the progressive ones) are applied to the whole personal income (not just to earnings).

Public Budget Constraint: $\sum T^{1}-\sum B^{1}+r \sum q C^{1}+\sum S^{1}=\sum T^{0}-\sum B^{0}+r \sum q C^{0}+\sum S^{0}$ where the superscript $\mathrm{R}$ denotes a generic reform and the superscript 0 denotes the current system. 


\section{Couples}

\section{GMI flat}

$C_{F}=\left\{\begin{array}{l}G / 2 \text { if } I_{F} \leq G / 2 \\ G / 2+\left(I_{F}-G / 2\right)(1-t) \text { if } I_{F}>G / 2\end{array}\right.$

$C_{M}=\left\{\begin{array}{l}G / 2 \text { if } I_{M} \leq G / 2 \\ G / 2+\left(I_{M}-G / 2\right)(1-t) \text { if } I_{M}>G / 2\end{array}\right.$

$T_{F}=\left\{\begin{array}{l}0 \text { if } I_{F} \leq G / 2 \\ \left(I_{F}-G / 2\right) t \text { if } I_{F}>G / 2\end{array}\right.$

$B_{F}=\left\{\begin{array}{l}G / 2-I_{F} \text { if } I_{F} \leq G / 2 \\ 0 \text { if } I_{F}>G / 2\end{array}\right.$

$T_{M}=\left\{\begin{array}{l}0 \text { if } I_{M} \leq G / 2 \\ \left(I_{M}-G / 2\right) t \text { if } I_{M}>G / 2\end{array}\right.$

$B_{M}=\left\{\begin{array}{l}G / 2-I_{M} \text { if } I_{M} \leq G / 2 \\ 0 \text { if } I_{M}>G / 2\end{array}\right.$

\section{GMI Progressive}

$$
\begin{aligned}
C_{F} & =\left\{\begin{array}{l}
G / 2 \text { if } I_{F} \leq G / 2 \\
G / 2+\varphi\left(I_{F}-G / 2\right) \text { if } I_{F}>G / 2
\end{array}\right. \\
C_{M} & =\left\{\begin{array}{l}
G / 2 \text { if } I_{M} \leq G / 2 \\
G / 2+\varphi\left(I_{M}-G / 2\right) \text { if } I_{M}>G / 2
\end{array}\right. \\
T_{F} & =\left\{\begin{array}{l}
0 \text { if } I_{F} \leq G / 2 \\
\left(I_{F}-G / 2\right)-\varphi\left(I_{F}-G / 2\right) \text { if } I_{F}>G / 2
\end{array}\right. \\
B_{F} & =\left\{\begin{array}{l}
G / 2-I_{F} \text { if } I_{F} \leq G / 2 \\
0 \text { if } I_{F}>G / 2
\end{array}\right. \\
T_{M} & =\left\{\begin{array}{l}
0 \text { if } I_{M} \leq G / 2 \\
\left(I_{M}-G / 2\right)-\varphi\left(I_{M}-G / 2\right) \text { if } I_{M}>G / 2
\end{array}\right. \\
B_{M} & =\left\{\begin{array}{l}
G / 2-I_{M} \text { if } I_{M} \leq G / 2 \\
0 \text { if } I_{M}>G / 2
\end{array}\right.
\end{aligned}
$$




\section{UBI Flat}

$$
\begin{aligned}
& C_{F}=G / 2+I_{M}(1-t) \\
& C_{M}=G / 2+I_{M}(1-t) \\
& T_{F}=t I_{M} \\
& B_{F}=G / 2 \\
& T_{M}=t I_{M} \\
& B_{M}=G / 2
\end{aligned}
$$

\section{UBI Progressive}

$$
\begin{aligned}
& C_{F}=G / 2+\varphi\left(I_{F}\right) \\
& C_{M}=G / 2+\varphi\left(I_{M}\right) \\
& T_{F}=I_{F}-\varphi\left(I_{F}\right) \\
& B_{F}=G / 2 \\
& T_{M}=I_{M}-\varphi\left(I_{M}\right) \\
& B_{M}=G / 2
\end{aligned}
$$

\section{WS Flat}

$C_{F}=\left\{\begin{array}{l}\left(I_{F}+\omega x_{F}\right) \text { if }\left(I_{F}+\omega x_{F}\right) \leq G / 2 \\ G / 2+\left(\left(I_{F}+\omega x_{F}\right)-G / 2\right)(1-t) \text { if }\left(I_{F}+\omega x_{F}\right)>G / 2\end{array}\right.$

$T_{F}=\left\{\begin{array}{l}0 \text { if }\left(I_{F}+\omega x_{F}\right) \leq G / 2 \\ \left(G / 2-\left(I_{F}+\omega x_{F}\right)\right) t \text { if }\left(I_{F}+\omega x_{F}\right)>G / 2\end{array}\right.$

$B_{F}=\omega g_{F}$

$C_{M}=\left\{\begin{array}{l}\left(I_{M}+\omega x_{M}\right) \text { if }\left(I_{M}+\omega x_{M}\right) \leq G / 2 \\ G / 2+\left(\left(I_{M}+\omega x_{M}\right)-G / 2\right)(1-t) \text { if }\left(I_{M}+\omega x_{M}\right)>G / 2\end{array}\right.$

$T_{M}=\left\{\begin{array}{l}0 \text { if }\left(I_{M}+\omega x_{M}\right) \leq G / 2 \\ \left(G / 2-\left(I_{M}+\omega x_{M}\right)\right) t \text { if }\left(I_{M}+\omega x_{M}\right)>G / 2\end{array}\right.$

$B_{M}=\omega x_{M}$ 


\section{WS Progressive}

$$
\begin{aligned}
& C_{F}=\left\{\begin{array}{l}
\left(I_{F}+\omega x_{F}\right) \text { if }\left(I_{F}+\omega x_{F}\right) \leq G / 2 \\
G / 2+\varphi\left(\left(I_{F}+\omega x_{F}\right)-G / 2\right) \text { if }\left(I_{F}+\omega x_{F}\right)>G / 2
\end{array}\right. \\
& T_{F}=\left\{\begin{array}{l}
0 \text { if }\left(I_{F}+\omega x_{F}\right) \leq G / 2 \\
\left(\left(I_{F}+\omega x_{F}\right)-G / 2\right)-\varphi\left(\left(I_{F}+\omega x_{F}\right)-G / 2\right) \text { if }\left(I_{F}+\omega x_{F}\right)>G / 2
\end{array}\right. \\
& B_{F}=\omega x_{F} \\
& C_{M}=\left\{\begin{array}{l}
\left(I_{M}+\omega x_{M}\right) \text { if }\left(I_{M}+\omega x_{M}\right) \leq G / 2 \\
G / 2+\varphi\left(\left(I_{M}+\omega x_{M}\right)-G / 2\right) \text { if }\left(I_{M}+\omega x_{M}\right)>G / 2
\end{array}\right. \\
& T_{M}=\left\{\begin{array}{l}
0 \text { if }\left(I_{M}+\omega x_{M}\right) \leq G / 2 \\
\left(\left(I_{M}+\omega x_{M}\right)-G / 2\right)-\varphi\left(\left(I_{M}+\omega x_{M}\right)-G / 2\right) \text { if }\left(I_{M}+\omega x_{M}\right)>G / 2
\end{array}\right. \\
& B_{M}=\omega x_{M}
\end{aligned}
$$

\section{GMI + WS Flat}

$C_{F}=\left\{\begin{array}{l}0.5 G / 2 \text { if }\left(I_{F}+\omega x_{F}\right) \leq 0.5 G / 2 \\ \left(I_{F}+\omega x_{F}\right) \text { if } 0.5 G / 2<\left(I_{F}+\omega x_{F}\right) \leq G / 2 \\ G / 2+\left(\left(I_{F}+\omega x_{F}\right)-G / 2\right)(1-t) \text { if }\left(I_{F}+\omega x_{F}\right)>G / 2\end{array}\right.$

$T_{F}=\left\{\begin{array}{l}0 \text { if }\left(I_{F}+\omega x_{F}\right) \leq G / 2 \\ \left(\left(I_{F}+\omega x_{F}\right)-G / 2\right) t \text { if }\left(I_{F}+\omega x_{F}\right)>G / 2\end{array}\right.$

$B_{F}=\left\{\begin{array}{l}\omega x_{F}+0.5 G / 2-\left(I_{F}+\omega x_{F}\right) \text { if }\left(I_{F}+\omega x_{F}\right) \leq 0.5 G / 2 \\ \omega x_{F} \text { if }\left(I_{F}+\omega x_{F}\right)>0.5 G / 2\end{array}\right.$

$C_{M}=\left\{\begin{array}{l}0.5 G / 2 \text { if }\left(I_{M}+\omega x_{M}\right) \leq 0.5 G / 2 \\ \left(I_{M}+\omega x_{M}\right) \text { if } 0.5 G / 2<\left(I_{M}+\omega x_{M}\right) \leq G / 2 \\ G / 2+\left(\left(I_{M}+\omega x_{M}\right)-G / 2\right)(1-t) \text { if }\left(I_{M}+\omega x_{M}\right)>G / 2\end{array}\right.$

$T_{M}=\left\{\begin{array}{l}0 \text { if }\left(I_{M}+\omega x_{M}\right) \leq G / 2 \\ \left(\left(I_{M}+\omega x_{M}\right)-G / 2\right) t \text { if }\left(I_{M}+\omega x_{M}\right)>G / 2\end{array}\right.$

$B_{M}=\left\{\begin{array}{l}\omega x_{M}+0.5 G / 2-\left(I_{M}+\omega x_{M}\right) \text { if }\left(I_{M}+\omega x_{M}\right) \leq 0.5 G / 2 \\ \omega x_{M} \text { if }\left(I_{M}+\omega x_{M}\right)>0.5 G / 2\end{array}\right.$ 


\section{GMI + WS Progressive}

$$
\begin{aligned}
& C_{F}=\left\{\begin{array}{l}
0.5 G / 2 \text { if }\left(I_{F}+\omega x_{F}\right) \leq 0.5 G / 2 \\
\left(I_{F}+\omega x_{F}\right) \text { if } 0.5 G / 2<\left(I_{F}+\omega x_{F}\right) \leq G / 2 \\
G / 2+\varphi\left(\left(I_{F}+\omega x_{F}\right)-G / 2\right) \text { if }\left(I_{F}+\omega x_{F}\right)>G / 2
\end{array}\right. \\
& T_{F}=\left\{\begin{array}{l}
0 \text { if }\left(I_{F}+\omega x_{F}\right) \leq G / 2 \\
\left(\left(I_{F}+\omega x_{F}\right)-G / 2\right)-\varphi\left(\left(I_{F}+\omega x_{F}\right)-G / 2\right) \text { if }\left(I_{F}+\omega x_{F}\right)>G / 2
\end{array}\right. \\
& B_{F}=\left\{\begin{array}{l}
\omega x_{F}+0.5 G / 2-\left(I_{F}+\omega x_{F}\right) \text { if }\left(I_{F}+\omega x_{F}\right) \leq 0.5 G / 2 \\
\omega x_{F} \text { if }\left(I_{F}+\omega x_{F}\right)>0.5 G / 2
\end{array}\right. \\
& C_{M}=\left\{\begin{array}{l}
0.5 G / 2 \text { if }\left(I_{M}+\omega g_{M}\right) \leq 0.5 G / 2 \\
\left(I_{M}+\omega x_{M}\right) \text { if } 0.5 G / 2<\left(I_{M}+\omega x_{M}\right) \leq G / 2 \\
G / 2+\varphi\left(\left(I_{M}+\omega x_{M}\right)-G / 2\right) \text { if }\left(I_{M}+\omega x_{M}\right)>G / 2
\end{array}\right. \\
& T_{M}=\left\{\begin{array}{l}
0 \text { if }\left(I_{M}+\omega x_{M}\right) \leq G / 2 \\
\left(\left(I_{M}+\omega x_{M}\right)-G / 2\right)-\varphi\left(\left(I_{M}+\omega x_{M}\right)-G / 2\right) \text { if }\left(I_{M}+\omega x_{M}\right)>G / 2
\end{array}\right. \\
& B_{M}=\left\{\begin{array}{l}
\omega x_{M}+0.5 G / 2-\left(I_{M}+\omega x_{M}\right) \text { if }\left(I_{M}+\omega x_{M}\right) \leq 0.5 G / 2 \\
\omega x_{M} \text { if }\left(I_{M}+\omega x_{M}\right)>0.5 G / 2
\end{array}\right.
\end{aligned}
$$




\section{UBI + WS Flat}

$C_{F}=\left\{\begin{array}{l}0.5 G / 2+\left(\mathrm{I}_{F}+w x_{F}\right) \text { if }\left(\mathrm{I}_{F}+w x_{F}\right) \leq 0.5 G / 2 \\ 0.5 G / 2+\left(I_{F}+w x_{F}\right)(1-t) \text { if }\left(I_{F}+w x_{F}\right)>0.5 G / 2\end{array}\right.$

$B_{F}=0.5 G / 2+w x_{F}$

$T_{F}=\left\{\begin{array}{l}0 \text { if }\left(\mathrm{I}_{F}+w x_{F}\right) \leq 0.5 G / 2 \\ t\left(I_{F}+w x_{F}\right) \text { if }\left(I_{F}+w x_{F}\right)>0\end{array}\right.$

$t\left(I_{F}+w x_{F}\right)$ if $\left(I_{F}+w x_{F}\right)>0.5 G / 2$

$C_{M}=\left\{\begin{array}{l}0.5 G / 2+\left(\mathrm{I}_{M}+w x_{M}\right) \text { if }\left(\mathrm{I}_{M}+w x_{M}\right) \leq 0.5 G / 2 \\ 0.5 G / 2+\left(I_{M}+w x_{M}\right)(1-t) \text { if }\left(I_{M}+w x_{M}\right)>0.5 G / 2\end{array}\right.$

$B_{M}=0.5 G / 2+w x_{M}$

$T_{M}=\left\{\begin{array}{l}0 \text { if }\left(\mathrm{I}_{M}+w x_{M}\right) \leq 0.5 G / 2 \\ t\left(I_{M}+w x_{M}\right) \text { if }\left(I_{M}+w x_{M}\right)>0.5 G / 2\end{array}\right.$

\section{UBI + WS Progressive}

$C_{F}=\left\{\begin{array}{l}0.5 G / 2+\left(\mathrm{I}_{F}+w x_{F}\right) \text { if }\left(\mathrm{I}_{F}+w x_{F}\right) \leq 0.5 G / 2 \\ 0.5 G / 2+\varphi\left(I_{F}+w x_{F}\right) \text { if }\left(I_{F}+w x_{F}\right)>0.5 G / 2\end{array}\right.$

$B_{F}=0.5 G / 2+w x_{F}$

$T_{F}=\left\{\begin{array}{l}0 \text { if }\left(\mathrm{I}_{F}+w x_{F}\right) \leq 0.5 G / 2 \\ \left(I_{F}+w x_{F}\right)-\varphi\left(I_{F}+w x_{F}\right) \text { if }\end{array}\right.$

$C_{M}=\left\{\begin{array}{l}0.5 G / 2+\left(\mathrm{I}_{M}+w x_{M}\right) \text { if }\left(\mathrm{I}_{M}+w x_{M}\right) \leq 0.5 G / 2 \\ 0.5 G / 2+\varphi\left(I_{M}+w x_{M}\right) \text { if }\left(I_{M}+w x_{M}\right)>0.5 G / 2\end{array}\right.$

$B_{M}=0.5 G / 2+w x_{M}$

$T_{M}=\left\{\begin{array}{l}0 \text { if }\left(\mathrm{I}_{M}+w x_{M}\right) \leq 0.5 G / 2 \\ \left(I_{M}+w x_{M}\right)-\varphi\left(I_{M}+w x_{M}\right) \text { if }\left(I_{M}+w x_{M}\right)>0.5 G / 2\end{array}\right.$ 
Singles

\section{GMI flat}

$C=\left\{\begin{array}{l}G \text { if } I \leq G \\ G+(I-G)(1-t) \text { if } I>G\end{array}\right.$

$T=\left\{\begin{array}{l}0 \text { if } I \leq G \\ (I-G) t \text { if } I>G\end{array}\right.$

$B=\left\{\begin{array}{l}G-I \text { if } I \leq G \\ 0 \text { if } I>G\end{array}\right.$

\section{GMI Progressive}

$C=\left\{\begin{array}{l}G \text { if } I \leq G \\ G+\varphi(I-G) \text { if } I>G\end{array}\right.$

$T=\left\{\begin{array}{l}0 \text { if } I \leq G \\ (I-G)-\varphi(I-G) \text { if } I>G\end{array}\right.$

$B=\left\{\begin{array}{l}G-I \text { if } I \leq G \\ 0 \text { if } I>G\end{array}\right.$

\section{UBI Flat}

$$
\begin{aligned}
& C=G+I(1-t) \\
& T=t I \\
& B=G
\end{aligned}
$$

\section{UBI Progressive}

$$
\begin{aligned}
& C=G+\varphi(I) \\
& T=I-\varphi(I) \\
& B=G
\end{aligned}
$$

\section{WS Flat}

$C=\left\{\begin{array}{l}(I+\omega x) \text { if }(I+\omega x) \leq G \\ G+((I+\omega x)-G)(1-t) \text { if }(I+\omega x)>G / 2\end{array}\right.$

$T=\left\{\begin{array}{l}0 \text { if }(I+\omega x) \leq G / 2 \\ (G-(I+\omega x)) t \text { if }(I+\omega x)>G\end{array}\right.$

$B=\omega g$ 


\section{WS Progressive}

$$
\begin{aligned}
& C=\left\{\begin{array}{l}
(I+\omega x) \text { if }(I+\omega x) \leq G \\
G+\varphi((I+\omega x)-G) \text { if }(I+\omega x)>G
\end{array}\right. \\
& T=\left\{\begin{array}{l}
0 \text { if }(I+\omega x) \leq G \\
((I+\omega x)-G)-\varphi((I+\omega x)-G) \text { if }(I+\omega x)>G
\end{array}\right. \\
& B=\omega x
\end{aligned}
$$

\section{GMI + WS Flat}

$C=\left\{\begin{array}{l}0.5 G \text { if }(I+\omega x) \leq 0.5 G \\ (I+\omega x) \text { if } 0.5 G<(I+\omega x) \leq G \\ G+((I+\omega x)-G)(1-t) \text { if }(I+\omega x)>G\end{array}\right.$

$T=\left\{\begin{array}{l}0 \text { if }(I+\omega x) \leq G \\ ((I+\omega x)-G) t \text { if }(I+\omega x)>G\end{array}\right.$

$B=\left\{\begin{array}{l}\omega x+0.5 G-(I+\omega x) \text { if }(I+\omega x) \leq 0.5 G \\ \omega x \text { if }(I+\omega x)>0.5 G\end{array}\right.$

\section{GMI + WS Progressive}

$$
\begin{aligned}
C & =\left\{\begin{array}{l}
0.5 G \text { if }(I+\omega x) \leq 0.5 G \\
(I+\omega x) \text { if } 0.5 G<(I+\omega x) \leq G \\
G+\varphi((I+\omega x)-G) \text { if }(I+\omega x)>G
\end{array}\right. \\
T & =\left\{\begin{array}{l}
0 \text { if }(I+\omega x) \leq G \\
((I+\omega x)-G)-\varphi((I+\omega x)-G) \text { if }(I+\omega x)>G
\end{array}\right. \\
B & =\left\{\begin{array}{l}
\omega x+0.5 G / 2-\left(I_{F}+\omega x\right) \text { if }\left(I_{F}+\omega x\right) \leq 0.5 G \\
\omega x \text { if }(I+\omega x)>0.5 G
\end{array}\right.
\end{aligned}
$$

\section{UBI + WS Flat}

$C=\left\{\begin{array}{l}0.5 G+(\mathrm{I}+w x) \text { if }(\mathrm{I}+w x) \leq 0.5 G \\ 0.5 G+(I+w x)(1-t) \text { if }(I+w x)>0.5 G\end{array}\right.$

$T=\left\{\begin{array}{l}0 \text { if }(\mathrm{I}+w x) \leq 0.5 G \\ t(I+w x) \text { if }(I+w x)>0.5 G\end{array}\right.$

$B=0.5 G+w x$ 


\section{UBI + WS Progressive}

$$
\begin{aligned}
& C=\left\{\begin{array}{l}
0.5 G+(\mathrm{I}+w x) \text { if }(\mathrm{I}+w x) \leq 0.5 G \\
0.5 G+\varphi\left(I_{F}+w x_{F}\right) \text { if }\left(I_{F}+w x_{F}\right)>0.5 G
\end{array}\right. \\
& T=\left\{\begin{array}{l}
0 \text { if }(\mathrm{I}+w x) \leq 0.5 G \\
(I+w x)-\varphi(I+w x) \text { if }(I+w x)>0.5 G
\end{array}\right. \\
& B=0.5 G+w x
\end{aligned}
$$




\section{References}

Aaberge, R. (2007) Gini's Nuclear Family, Journal of Economic Inequality, 5 (3), 305-322.

Aaberge, R. and U. Colombino (2011a) Empirical Optimal Income Taxation: A Microeconometric Application to Norway, Working Paper CHILD n. 16/2011.

Aaberge, R. and U. Colombino (2011b) Designing Optimal Taxes with a Microeconometric Model of Labour Supply, Scandinavian Journal of Economics, forthcoming.

Aaberge, R., J.K. Dagsvik and S. Strøm (1995) Labour Supply Responses and Welfare Effects of Tax Reforms, Scandinavian Journal of Economics, 97 (4), 635-659.

Aaberge, R., U. Colombino and S. Strøm (1999) Labor Supply in Italy: An Empirical Analysis of Joint Household Decisions, with Taxes and Quantity Constraints, Journal of Applied Econometrics, 14 (4), 403-422.

Aaberge, R., U. Colombino and S. Strøm (2000) Labour supply responses and welfare effects from replacing current tax rules by a flat tax: empirical evidence from Italy, Norway and Sweden, Journal of Population Economics, 13 (4), 595621.

Aaberge, R., U. Colombino and S. Strøm (2004) Do More Equal Slices Shrink the Cake? An Empirical Evaluation of Tax-Transfer Reform Proposals in Italy, Journal of Population Economics, 17 (4), pp. 767-785.

Aaberge, R., U. Colombino and T. Wennemo (2002) Heterogeneity in the elasticity of labour supply in Italy and some policy implications, Working Paper CHILD n. 21/2002.

Aaberge, R., U. Colombino and T. Wennemo (2009) Evaluating Alternative Representations of the Choice Sets in Models of Labour Supply, Journal of Economic Surveys, 23 (3), 586-612.

Atkinson, A. B. (1987) On the measurement of poverty, Econometrica, 55, 749-764.

Atkinson, B. A. (1995) Public Economics in Action, Clarendon Press, Oxford.

Atkinson, B. A. (1996) The Case for Participation Income, The Political Quarterly, 3, 67-70.

Atkinson, B. A. (2002) How Basic Income is Moving up the Policy Agenda: News from the Future, Paper presented at the $9^{\text {th }}$ BIEN International Congress, 13 September 2002, Geneva (revised version).

Barrientos, A. and P. Lloyd-Sherlock (2002) Non-Contributory Pensions and Social Protection, Discussion paper no. 12, Issues in Social Protection, Geneva: ILO.

Ben-Akiva, M. and S. R. Lerman (1985) Discrete Choice Analysis, Cambridge (Ma.), The MIT Press.

Blundell, R., M. Brewer, P. Haan and A. Shephard (2009) Optimal income taxation of lone mothers: an empirical comparison for Britain and Germany, Economic Journal, Vol. 119 (535), F101-F121.

Bourguignon, F. and A. Spadaro (2006) Microsimulation as a Tool for Evaluating Redistribution Policies, ECINEQ 200620. 
Busilacchi, G. (2008) The different regimes of minimum income policies in the enlarged Europe, Proceedings of BIEN $12^{\text {th }}$ International Conference, 20-21 June 2008, Dublin.

Colombino, U. (1998) Evaluating the effects of new telephone tariffs on residential users' demand and welfare. A model for Italy, Information Economics and Policy, 10 (3), 283-303.

Colombino, U. (2010) Equilibrium policy simulations with random utility models of labour supply, IZA Discussion Paper No. 5262.

Colombino, U., M. Locatelli, E., Narazani and C. O'Donoghue (2010) Alternative Basic Income Mechanisms: An Evaluation Exercise with a Microeconometric Model, Basic Income Studies, 5(1).

Creedy, J. and A. Duncan (2001) Aggregating Labour Supply and Feedback Effects in Microsimulation, IFS WP01/24.

Creedy, J. and G. Kalb (2005) Discrete Hours Labour Supply Modelling: Specification, Estimation and Simulation, Journal of Economic Surveys, 19 (5), 697-734.

Dagsvik, J. (2000) Aggregation in Matching Markets, International Economic Review, 41(1), 27-57.

Dagsvik, J. (1994) Discrete and Continuous Choice, Max-Stable Processes, and Independence from Irrelevant Attributes, Econometrica, 62(5), 1179-1205.

Dagsvik, J. and S. Strøm (2006) Sectoral Labour Supply, Choice Restrictions and Functional Form, Journal of Applied Econometrics 21(6), 803-826.

De Vincenti, C. And R. Paladini (2009) Personal Income Tax Design for Italy: Lessons from the Theory, Rivista Italiana degli Economisti, XIV, 1, 7-46.

Diamond, P. (1998) Optimal Income Taxation An Example with a U-Shaped pattern of Optimal Marginal Tax Rates, American Economic Review, 88, 83-95.

Diamond, P. and E. Saez (2011) The Case for a Progressive Tax: From Basic Research to Policy Recommendations, CesIfo WP No. 3548.

Duncan, A. and C. Giles (1996) Labour supply incentives and recent Family Credit reforms, Economic Journal, 106 (434), 142-155.

Haan, P. and K. Wrohlich (2007) Optimal Taxation: The Design of Child Related Cash- and In-Kind-Benefits, IZA Discussion Papers 3128.

Kalb, G. (2000) Accounting for Involuntary Unemployment and the Cost of Part-Time Work, Working Paper No. BP-35, Centre of Policy Studies, Monash University, Australia.

Kornstad, T. and T. Thoresen (2007) A Discrete Choice Model for Labor Supply and Childcare, Journal of Population Economics, 20(4), 781-803.

King, M. (1983) Welfare Analysis of Tax Reforms Using Household Data, Journal of Public Economics, 21, 183-214.

Friedman, M. (1962) Capitalism and Freedom, Chicago, University of Chicago Press. 
Gruber, J. and E. Saez (2002) The Elasticity of Taxable Income: Evidence and Implications, Journal of Public Economics, 84: 1-32.

Immervoll, H., H. J. Kleven, C. T. Kreiner and E. Saez (2007) Welfare Reforms in European Countries: A Microsimulation Analysis, Economic Journal, 117 (1), 1-44.

McFadden, D. (1974) Measurement of Urban Travel Demand, Journal of Public Economics, 3 (4), 303-328.

McFadden, D. (1978) Modelling the Choice of Residential Location, in A. Karlquist, L. Lundquist, F. Snickard and J.J. Weilbull (eds.): Spatial Interaction Theory and Planning Models, Amsterdam, North-Holland.

Meade, J. (1972) Poverty in the Welfare State, Oxford Economic Papers, (new series), 24 (3), 289-326.

Meghir, C. and D. Phillips (2008) Labour Supply and Taxes, Institute for Fiscal Studies, WP 08/04.

Mirlees, J. A. (1971) An Exploration in the Theory of Optimal Income Taxation, Review of Economic Studies, 38 (2), $175-208$.

Røed, K. and S. Strøm (2002) Progressive Taxes and the Labour Market - Is the Trade-Off between Equality and Efficiency Inevitable?, Journal of Economic Surveys, 16 (1), pp. 77-110.

Saez, E. (2001) Using Elasticities to Derive Optimal Income Tax Rates, Review of Economic Studies, 68, 205-229.

Saez, E. (2002) Optimal income transfer programs: intensive versus extensive labor supply Responses, Quarterly Journal of Economics, 117 (3), 1039-1073.

Sen, A. (1974) Information Bases of Alternative Welfare Approaches: Aggregation and Income Distribution, Journal of Public Economics, 3 (4), 387-403.

Sen, A. (1976) Real National Income, Review of Economic Studies, 43 (1), 19-39.

Standing, G. (2008) How Cash Transfers Promote the Case for Basic Income, Basic Income Studies, 3 (1), Article 5.

Van Parijs, P. (1995) Real Freedom for All, Oxford, Oxford University Press.

Van Soest, A. (1995) Structural models of family labour supply, Journal of Human Resources, 30 (1), 63-88.

Van Trier, W. (1995) Every One a King, Department of Sociology, Katholieke Universiteit Leuven, Leuven. 